\title{
A mixed finite element method for the generalized Stokes problem*
}

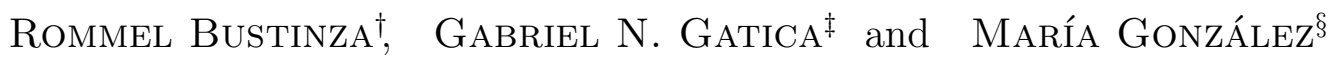

\begin{abstract}
We present and analyse a new mixed finite element method for the generalized Stokes problem. The approach, which is a natural extension of a previous procedure applied to quasiNewtonian Stokes flows, is based on the introduction of the flux and the tensor gradient of the velocity as further unknowns. This yields a two-fold saddle point operator equation as the resulting variational formulation. Then, applying a slight generalization of the well known Babuška-Brezzi theory, we prove that the continuous and discrete formulations are well posed, and derive the associated a priori error analysis. In particular, the finite element subspaces providing stability coincide with those employed for the usual Stokes flows except for one of them that needs to be suitably enriched. We also develop an a-posteriori error estimate (based on local problems) and propose the associated adaptive algorithm to compute the finite element solutions. Several numerical results illustrate the performance of the method and its capability to localize boundary layers, inner layers, and singularities.
\end{abstract}

\section{Introduction}

The generalized Stokes problem, which is a Stokes-like linear system with a dominating zeroth order term, arises naturally in the time discretisation of the corresponding non-steady equations, and hence it plays a fundamental role in the numerical simulation of viscous incompressible flows (laminar and turbulent). Indeed, the most expensive part of the solution procedure for the timedependent Navier-Stokes equations reduces to solving the generalized Stokes problem at each nonlinear iteration. In order to define it explicitly, we first let $\Omega$ be a bounded open subset of $\mathbb{R}^{2}$ with Lipschitz continuous boundary $\Gamma$. Then, given $\mathbf{f} \in\left[L^{2}(\Omega)\right]^{2}$ and $\mathbf{g} \in\left[H^{1 / 2}(\Gamma)\right]^{2}$, we look for the velocity $\mathbf{u}:=\left(u_{1}, u_{2}\right)^{\mathrm{t}}$ and the pressure $p$ of a fluid occupying the region $\Omega$, such that

$$
\begin{aligned}
\alpha \mathbf{u}-\nu \Delta \mathbf{u}+\nabla p & =\mathbf{f} & & \text { in } \Omega, \\
\operatorname{div}(\mathbf{u}) & =0 & & \text { in } \Omega, \\
\mathbf{u} & =\mathbf{g} & & \text { on } \Gamma,
\end{aligned}
$$

where $\nu$ is a positive constant called kinematic viscosity of the fluid and $\alpha$ is a positive parameter proportional to the inverse of the time-step. Throughout the rest of the paper we assume that $\alpha \geq \nu$. Also, we remark that, as a consequence of the incompressibility of the fluid, the Dirichlet

\footnotetext{
${ }^{*}$ This research was partially supported by CONICYT-Chile through the FONDAP Program in Applied Mathematics, and by the Dirección de Investigación of the Universidad de Concepción through the Advanced Research Groups Program.

${ }^{\dagger}$ Departamento de Ingeniería Matemática, Universidad de Concepción, Casilla 160-C, Concepción, Chile, email: rbustinz@ing-mat.udec.cl

${ }^{\ddagger} \mathbf{G I}^{2}$ MA, Departamento de Ingeniería Matemática, Universidad de Concepción, Casilla 160-C, Concepción, Chile, e-mail: ggatica@ing-mat.udec.cl

${ }^{\S}$ Departamento de Matemáticas, Universidade da Coruña, 15071 A Coruña, España, e-mail: mgtaboad@udc.es
} 
datum $\mathbf{g}$ must satisfy the compatibility condition $\int_{\Gamma} \mathbf{g} \cdot \mathbf{n} d s=0$, where $\mathbf{n}$ is the unit outward normal to $\Gamma$.

In recent years considerable effort has gone into the design and study of efficient numerical methods to solve (1.1). The new proposed algorithms apply and combine different techniques, which include Uzawa's schemes, splittings of boundary conditions, fictitious domains, domain decomposition, stabilization, and preconditioning (see, e.g. [2], [4], [7], [8], [16], [19], [21], and the references therein). A common feature to these papers is that they all deal with the usual pressure-velocity variational formulation of the problem, in which the unknowns live in $L^{2}(\Omega)$ and $H^{1}(\Omega)$, respectively. In particular, this means that the finite element subspace for the velocity needs to be a subset of the continuous functions. In addition, the Dirichlet boundary condition, being essential and non-homogeneous, cannot be incorporated either in the continuous and discrete formulations or in the definitions of the spaces involved, and therefore one is necessarily led to a non-conforming Galerkin scheme. Certainly, the latter concern refers to the theoretical analysis of the method since the interpolation of essential boundary conditions causes no problems in the practical implementation of the corresponding Galerkin scheme.

On the other hand, within a dual-mixed setting the velocity becomes an unknown in $L^{2}(\Omega)$, which gives more flexibility to choose the associated finite element subspace (for instance, piecewise constant functions become a feasible choice). Furthermore, the Dirichlet boundary condition, being now natural, is incorporated directly into the right hand sides (linear functionals) of the continuous and discrete formulations, and hence the error analysis arising from a nonconforming scheme is avoided. Another important advantage of using dual-mixed methods lies on the possibility of introducing further unknowns with a physical interest (for instance, the flux). These unknowns are then approximated directly, which avoids any numerical postprocessing yielding additional sources of error.

As a recent example of the above, we recall here that in [12] and [13] we introduce and analyse a dual-mixed formulation for a class of quasi-Newtonian Stokes flows whose kinematic viscosities are nonlinear monotone functions of the gradient of the velocity. The mixed finite element method proposed there simply relies on the introduction of the flux and the tensor gradient of the velocity as auxiliary unknowns, which yields a two-fold saddle point operator equation as the resulting variational formulation. Therefore, the abstract theory developed in [11], which is a slight generalization of the well known Babuška-Brezzi theory, is applied to prove that the continuous and discrete schemes are well posed. In particular, it is shown that the stability of the Galerkin scheme only requires low-order finite element subspaces: it suffices to use Raviart-Thomas spaces of order zero to approximate the flux and piecewise constant functions to approximate the other unknowns. In addition, since the monotonicity certainly includes the linear case, we also obtain as a by-product a new mixed finite element method for the linear Stokes equation (problem (1.1) with $\alpha=0$ ).

Up to the authors' knowledge, there are no dual-mixed methods available in the current literature for the generalized Stokes problem. This gap is somehow filled by the present work, and hence we believe this is one of the main motivations for it. More precisely, the purpose of this paper is to extend the analysis from [12] and [13] to our problem (1.1) with moderately large values of the parameter $\alpha$, which includes, similarly as we did in [13], the derivation of aposteriori error estimates based on local problems. The rest of the paper is organized as follows. In Section 2 we derive the continuous dual-mixed variational formulation of problem (1.1) and prove that it is well posed. Then, in Section 3 we present and analyse the corresponding mixed finite element scheme. Again, we use Raviart-Thomas spaces of order zero to approximate the flux and piecewise constant functions to approximate the velocity and the pressure. However, in 
order to guarantee the stability of the Galerkin scheme, we need to include in the approximation space of the tensor gradient of the velocity the deviator of the vector Raviart-Thomas space of order zero. In this way, we prove that the discrete scheme has a unique solution and derive quasi-optimal error estimates and the corresponding rates of convergence. Next, in Section 4 we develop an implicit reliable and quasi-efficient a posteriori error estimate, and a fully explicit reliable one, and propose the adaptive algorithm associated to the latter to compute the finite element solutions. Finally, several numerical results are reported in Section 5.

In what follows, given any Hilbert space $H$, we denote by $H^{2}$ and $H^{2 \times 2}$ the spaces of vectors and tensors of order two, respectively, with entries in $H$, provided with the product norms induced by the norm of $H$. In addition, for any $\boldsymbol{\tau}:=\left(\tau_{i j}\right), \boldsymbol{\zeta}:=\left(\zeta_{i j}\right) \in \mathbb{R}^{2 \times 2}$, we denote $\operatorname{tr}(\boldsymbol{\tau}):=\tau_{11}+\tau_{22}$ and $\boldsymbol{\tau}: \boldsymbol{\zeta}:=\sum_{i, j=1}^{2} \tau_{i j} \zeta_{i j}$. The deviator of tensor $\boldsymbol{\tau}$ is denoted by $\operatorname{dev}(\boldsymbol{\tau}):=\boldsymbol{\tau}-\frac{1}{2} \operatorname{tr}(\boldsymbol{\tau}) \mathbf{I}$. We remark that $\operatorname{tr}(\operatorname{dev}(\boldsymbol{\tau}))=0$.

\section{The continuous variational formulation}

We first proceed as in [12] and introduce two additional unknowns in $\Omega$, namely, the tensor gradient of the velocity $\mathbf{t}:=\nabla \mathbf{u}$ and the flux $\boldsymbol{\sigma}:=\nu \nabla \mathbf{u}-p \mathbf{I}$, where $\mathbf{I}$ is the identity matrix in $\mathbf{R}^{2 \times 2}$. It follows that the equilibrium equation becomes

$$
\alpha \mathbf{u}-\operatorname{div}(\boldsymbol{\sigma})=\mathbf{f} \text { in } \Omega,
$$

where $\boldsymbol{\sigma}:=\nu \mathbf{t}-p \mathbf{I}$ and $\mathbf{d i v}$ denotes the vector divergence operator. In addition, since $\operatorname{div}(\mathbf{u})=$ $\operatorname{tr}(\mathbf{t})$ in $\Omega$, we can rewrite the incompressibility condition as

$$
\operatorname{tr}(\mathbf{t})=0 \quad \text { in } \Omega .
$$

Now, multiplying the relation $\mathbf{t}=\nabla \mathbf{u}$ by a tensor $\boldsymbol{\tau}$, integrating by parts and using that $\mathbf{u}=\mathbf{g}$ on $\Gamma$, we get:

$$
\int_{\Omega} \boldsymbol{\tau}: \mathbf{t}+\int_{\Omega} \operatorname{div}(\boldsymbol{\tau}) \cdot \mathbf{u}=\langle\boldsymbol{\tau} \mathbf{n}, \mathbf{g}\rangle \quad \forall \boldsymbol{\tau} \in H(\operatorname{div} ; \Omega) .
$$

Hereafter, $\langle\cdot, \cdot\rangle$ denotes the duality pairing of $\left[H^{-1 / 2}(\Gamma)\right]^{2}$ and $\left[H^{1 / 2}(\Gamma)\right]^{2}$ with $\left[L^{2}(\Gamma)\right]^{2}$ as pivot space, and $H(\operatorname{div} ; \Omega)$ is the space of tensors $\boldsymbol{\tau} \in\left[L^{2}(\Omega)\right]^{2 \times 2}$ satisfying $\operatorname{div}(\boldsymbol{\tau}) \in\left[L^{2}(\Omega)\right]^{2}$. We also recall that $H(\operatorname{div} ; \Omega)$, endowed with the inner product $\langle\boldsymbol{\zeta}, \boldsymbol{\tau}\rangle_{H(\operatorname{div} ; \Omega)}:=\langle\boldsymbol{\zeta}, \boldsymbol{\tau}\rangle_{\left[L^{2}(\Omega)\right]^{2 \times 2}}+$ $\langle\operatorname{div} \boldsymbol{\zeta}, \operatorname{div} \boldsymbol{\tau}\rangle_{\left[L^{2}(\Omega)\right]^{2}}$, is a Hilbert space, where $\langle\cdot, \cdot\rangle_{\left[L^{2}(\Omega)\right]^{2 \times 2}}$ and $\langle\cdot, \cdot\rangle_{\left[L^{2}(\Omega)\right]^{2}}$ stand for the usual inner products of $\left[L^{2}(\Omega)\right]^{2 \times 2}$ and $\left[L^{2}(\Omega)\right]^{2}$, respectively.

Then, testing the relation $\boldsymbol{\sigma}=\nu \mathbf{t}-p \mathbf{I}$, the equations (2.1) and (2.2), and reordering appropriately the resulting equations and (2.3), we obtain the following mixed variational formulation: Find $(\mathbf{t}, \mathbf{u}, \boldsymbol{\sigma}, p) \in\left[L^{2}(\Omega)\right]^{2 \times 2} \times\left[L^{2}(\Omega)\right]^{2} \times H(\operatorname{div} ; \Omega) \times L^{2}(\Omega)$ such that

$$
\begin{array}{rrrr}
\nu \int_{\Omega} \mathbf{t}: \mathbf{s} & -\int_{\Omega} \boldsymbol{\sigma}: \mathbf{s} & -\int_{\Omega} p \operatorname{tr}(\mathbf{s}) & = \\
& \alpha \int_{\Omega} \mathbf{u} \cdot \mathbf{v}-\int_{\Omega} \operatorname{div}(\boldsymbol{\sigma}) \cdot \mathbf{v} & & =\int_{\Omega} \mathbf{f} \cdot \mathbf{v}, \\
-\int_{\Omega} \boldsymbol{\tau}: \mathbf{t}-\int_{\Omega} \operatorname{div}(\boldsymbol{\tau}) \cdot \mathbf{u} & & -\langle\boldsymbol{\tau} \mathbf{n}, \mathbf{g}\rangle, \\
-\int_{\Omega} q \operatorname{tr}(\mathbf{t}) & = & 0,
\end{array}
$$


for all $(\mathbf{s}, \mathbf{v}, \boldsymbol{\tau}, q) \in\left[L^{2}(\Omega)\right]^{2 \times 2} \times\left[L^{2}(\Omega)\right]^{2} \times H(\operatorname{div} ; \Omega) \times L^{2}(\Omega)$.

Next, we observe that the variational formulation (2.4) is not uniquely solvable since given any solution $(\mathbf{t}, \mathbf{u}, \boldsymbol{\sigma}, p)$ of this problem and any $c \in \mathbb{R},(\mathbf{t}, \mathbf{u}, \boldsymbol{\sigma}+c \mathbf{I}, p-c)$ also becomes a solution. Therefore, in order to guarantee uniqueness we proceed as in [5] and require that $\int_{\Omega} \operatorname{tr}(\boldsymbol{\sigma})=0$, which leads to the introduction of a new unknown, a Lagrange multiplier $\xi \in \mathbb{R}$. Thus, from now on we consider the following mixed variational formulation of (1.1): Find $(\mathbf{t}, \mathbf{u}, \boldsymbol{\sigma}, p, \xi) \in\left[L^{2}(\Omega)\right]^{2 \times 2} \times\left[L^{2}(\Omega)\right]^{2} \times H(\operatorname{div} ; \Omega) \times L^{2}(\Omega) \times \mathbb{R}$ such that

$$
\begin{aligned}
& \nu \int_{\Omega} \mathbf{t}: \mathbf{s} \quad-\int_{\Omega} \boldsymbol{\sigma}: \mathbf{s} \quad-\int_{\Omega} p \operatorname{tr}(\mathbf{s})=0, \\
& \alpha \int_{\Omega} \mathbf{u} \cdot \mathbf{v}-\int_{\Omega} \operatorname{div}(\sigma) \cdot \mathbf{v} \quad=\int_{\Omega} \mathbf{f} \cdot \mathbf{v}, \\
& -\int_{\Omega} \boldsymbol{\tau}: \mathbf{t} \quad-\int_{\Omega} \operatorname{div}(\tau) \cdot \mathbf{u} \\
& \begin{aligned}
+\xi \int_{\Omega} \operatorname{tr}(\boldsymbol{\tau}) & =-\langle\boldsymbol{\tau} \mathbf{n}, \mathbf{g}\rangle, \\
& =0 \\
\eta \int_{\Omega} \operatorname{tr}(\boldsymbol{\sigma}) & =0 .
\end{aligned} \\
& -\int_{\Omega} q \operatorname{tr}(\mathbf{t})
\end{aligned}
$$

for all $(\mathbf{s}, \mathbf{v}, \boldsymbol{\tau}, q, \eta) \in\left[L^{2}(\Omega)\right]^{2 \times 2} \times\left[L^{2}(\Omega)\right]^{2} \times H(\operatorname{div} ; \Omega) \times L^{2}(\Omega) \times \mathbb{R}$. We remark here that taking $\boldsymbol{\tau}=\mathbf{I}$ in the third equation and applying (2.2) and the compatibility condition for the Dirichlet data, we find a priori that $\xi=0$. However, we keep this artificial unknown to ensure the symmetry and the well-posedness of the whole formulation.

In order to prove the unique solvability of the variational formulation (2.5), we write it now as a system of operator equations with a two-fold saddle point structure. To this end, we first define the spaces $X_{1}:=\left[L^{2}(\Omega)\right]^{2 \times 2} \times\left[L^{2}(\Omega)\right]^{2}, M_{1}:=H(\operatorname{div} ; \Omega)$, and $M:=L^{2}(\Omega) \times \mathbb{R}$. Then, we introduce the operators and functionals $\mathbf{A}_{1}: X_{1} \rightarrow X_{1}^{\prime}, \mathbf{B}_{1}: X_{1} \rightarrow M_{1}^{\prime}, \mathbf{B}^{p}: X_{1} \rightarrow L^{2}(\Omega)$, $\mathbf{B}^{\xi}: M_{1} \rightarrow \mathbb{R}, \mathbf{F}_{1} \in X_{1}^{\prime}$, and $\mathbf{F}_{2} \in M_{1}^{\prime}$, as suggested by the structure of (2.5), so that this problem can be stated as: Find $((\mathbf{t}, \mathbf{u}), \boldsymbol{\sigma},(p, \xi)) \in X_{1} \times M_{1} \times M$ such that

$$
\begin{aligned}
& {\left[\mathbf{A}_{1}(\mathbf{t}, \mathbf{u}),(\mathbf{s}, \mathbf{v})\right]+\left[\mathbf{B}_{1}(\mathbf{s}, \mathbf{v}), \boldsymbol{\sigma}\right]+\left[\mathbf{B}^{p}(\mathbf{s}, \mathbf{v}), p\right]=\left[\mathbf{F}_{1},(\mathbf{s}, \mathbf{v})\right],} \\
& {\left[\mathbf{B}_{1}(\mathbf{t}, \mathbf{u}), \boldsymbol{\tau}\right]+\left[\mathbf{B}^{\xi}(\boldsymbol{\tau}), \xi\right]=\left[\mathbf{F}_{2}, \boldsymbol{\tau}\right],} \\
& {\left[\mathbf{B}^{p}(\mathbf{t}, \mathbf{u}), q\right]+\left[\mathbf{B}^{\xi}(\boldsymbol{\sigma}), \eta\right] \quad=0,}
\end{aligned}
$$

for all $((\mathbf{s}, \mathbf{v}), \boldsymbol{\tau},(q, \eta)) \in X_{1} \times M_{1} \times M$, where $[\cdot, \cdot]$ denotes the duality pairing induced by the operators and functionals used in each case.

We now let $X:=X_{1} \times M_{1}$, identify $X^{\prime}$ with $X_{1}^{\prime} \times M_{1}^{\prime}$, and define $\mathbf{A}: X \rightarrow X^{\prime}$ as the matrix operator

$$
\mathbf{A}:=\left[\begin{array}{cc}
\mathbf{A}_{1} & \mathbf{B}_{1}^{\prime} \\
\mathbf{B}_{1} & \mathbf{O}
\end{array}\right]
$$

where $\mathbf{B}_{1}^{\prime}: M_{1} \rightarrow X_{1}^{\prime}$ is the adjoint of $\mathbf{B}_{1}$, and $\mathbf{O}$ denotes, from now on, a generic null operator/functional. Hence, (2.6) can be set equivalently as: Find $((\mathbf{t}, \mathbf{u}, \boldsymbol{\sigma}),(p, \xi)) \in X \times M$ such that

$$
\left[\begin{array}{ll}
\mathbf{A} & \mathbf{B}^{\prime} \\
\mathbf{B} & \mathbf{O}
\end{array}\right]\left[\begin{array}{c}
(\mathbf{t}, \mathbf{u}, \boldsymbol{\sigma}) \\
(p, \xi)
\end{array}\right]=\left[\begin{array}{c}
\mathbf{F} \\
\mathbf{O}
\end{array}\right]
$$

where $\mathbf{B}: X \rightarrow M$ is defined by $[\mathbf{B}(\mathbf{r}, \mathbf{w}, \boldsymbol{\zeta}),(q, \eta)]:=\left[\mathbf{B}^{p}(\mathbf{r}, \mathbf{w}), q\right]+\left[\mathbf{B}^{\xi}(\boldsymbol{\zeta}), \eta\right], \mathbf{B}^{\prime}: M \rightarrow X^{\prime}$ is the adjoint of $\mathbf{B}$, and $\mathbf{F} \in X^{\prime}$ is defined by $[\mathbf{F},(\mathbf{s}, \mathbf{v}, \boldsymbol{\tau})]:=\left[\mathbf{F}_{1},(\mathbf{s}, \mathbf{v})\right]+\left[\mathbf{F}_{2}, \boldsymbol{\tau}\right]$ for all 
$(\mathbf{s}, \mathbf{v}, \boldsymbol{\tau}),(\mathbf{r}, \mathbf{w}, \boldsymbol{\zeta}) \in X$ and for all $(q, \eta) \in M$. In this way, the two-fold saddle point structure of (2.6) becomes clear from (2.7) and (2.8) since $\mathbf{A}$ itself has the saddle point structure.

We now apply the abstract theory from [11] (see also the related results given in [10] and [14]) to establish the solvability and continuous dependence of (2.6).

TheOREM 2.1 Problem (2.6) has a unique solution $((\mathbf{t}, \mathbf{u}), \boldsymbol{\sigma},(p, \xi)) \in X_{1} \times M_{1} \times M$. Moreover, there exists a positive constant $C(\alpha, \nu)=O\left(\frac{\alpha^{3}}{\nu}\right)$, independent of the solution, such that

$$
\|((\mathbf{t}, \mathbf{u}), \boldsymbol{\sigma},(p, \xi))\|_{X_{1} \times M_{1} \times M} \leq C(\alpha, \nu)\left\{\left\|\mathbf{F}_{1}\right\|+\left\|\mathbf{F}_{2}\right\|\right\} .
$$

Proof. We observe first that the operators $\mathbf{A}_{1}, \mathbf{B}_{1}$ and $\mathbf{B}$ are all linear and bounded. In particular, it is easy to see that $\left\|\mathbf{A}_{1}\right\|=O(\alpha)$ and that both $\left\|\mathbf{B}_{1}\right\|$ and $\|\mathbf{B}\|$ are of $O(1)$. In addition, since $\alpha \geq \nu$, we deduce that $\mathbf{A}_{1}$ is $X_{1}$-elliptic with ellipticity constant $\nu$. Thus, according to the linear version of Theorem 2.4 in [11] (see also Theorem 2 in [10]), it only remains to show that $\mathbf{B}$ and $\mathbf{B}_{1}$ satisfy the corresponding inf-sup conditions on $X \times M$ and on the kernel of $\mathbf{B}$, respectively.

Indeed, given $(q, \eta) \in M$ we get lower bounds for $\sup _{(\mathbf{s}, \mathbf{v}, \boldsymbol{\tau}) \in X \backslash\{0\}} \frac{[\mathbf{B}(\mathbf{s}, \mathbf{v}, \boldsymbol{\tau}),(q, \eta)]}{\|(\mathbf{s}, \mathbf{v}, \boldsymbol{\tau})\|_{X}}$ by taking $(\mathbf{s}, \mathbf{v}, \boldsymbol{\tau})=(0,0, \eta \mathbf{I})$ and $(\mathbf{s}, \mathbf{v}, \boldsymbol{\tau})=(-q \mathbf{I}, 0,0)$, which yields the inf-sup condition for $\mathbf{B}$.

Next, we realize that the null space of the operator $\mathbf{B}$ is $\tilde{X}=\tilde{X}_{1} \times \tilde{M}_{1}$, where $\tilde{X}_{1}:=$ $\left\{(\mathbf{s}, \mathbf{v}) \in X_{1}: \operatorname{tr}(\mathbf{s})=0\right.$ in $\left.\Omega\right\}$ and $\tilde{M}_{1}:=\left\{\boldsymbol{\tau} \in M_{1}: \int_{\Omega} \operatorname{tr}(\boldsymbol{\tau})=0\right\}$. Thus, given $\boldsymbol{\tau} \in \tilde{M}_{1}$ we get now lower bounds for $\sup _{(\mathbf{s}, \mathbf{v}) \in \tilde{X}_{1} \backslash\{0\}} \frac{\left[\mathbf{B}_{1}(\mathbf{s}, \mathbf{v}), \boldsymbol{\tau}\right]}{\|(\mathbf{s}, \mathbf{v})\|_{X_{1}}}$ by taking $(\mathbf{s}, \mathbf{v})=(\mathbf{0},-\operatorname{div}(\boldsymbol{\tau}))$ and $(\mathbf{s}, \mathbf{v})=(-\operatorname{dev}(\boldsymbol{\tau}), \mathbf{0})$, which, using Lemma 3.1 in [1], yields the inf-sup condition for $\mathbf{B}_{1}$. This lower bound is also obtained when $\operatorname{div}(\boldsymbol{\tau})=0$ or $\operatorname{dev}(\boldsymbol{\tau})=\mathbf{0}$. We omit details.

Finally, we remark that the order of the continuous dependence constant $C(\alpha, \nu)$ follows from the analysis provided in Section 2 of [11] and from a particular case of Proposition 2.3 in $[22]$.

\section{The mixed finite element scheme}

In what follows we assume, for simplicity, that $\Gamma$ is a polygonal curve. Then, we let $\left\{\mathcal{T}_{h}\right\}_{h>0}$ be a regular family of triangulations of $\bar{\Omega}$ by triangles $T$ of diameter $h_{T}$ such that $h:=\max \left\{h_{T}: T \in\right.$ $\left.\mathcal{T}_{h}\right\}$ and $\bar{\Omega}=\cup\left\{T: T \in \mathcal{T}_{h}\right\}$. Also, we let $X_{1, h}^{\mathbf{t}}, X_{1, h}^{\mathbf{u}}, M_{1, h}$, and $M_{h}^{p}$ be finite element subspaces for the unknowns t $\mathbf{t}, \mathbf{u}, \boldsymbol{\sigma}$, and $p$, respectively, and define $X_{1, h}:=X_{1, h}^{\mathbf{t}} \times X_{1, h}^{\mathbf{u}}$ and $M_{h}:=M_{h}^{p} \times \mathbb{R}$. Then, the Galerkin scheme associated with problem (2.6) reads: Find $\left(\left(\mathbf{t}_{h}, \mathbf{u}_{h}\right), \boldsymbol{\sigma}_{h},\left(p_{h}, \xi_{h}\right)\right) \in$ $X_{1, h} \times M_{1, h} \times M_{h}$ such that

$$
\begin{aligned}
& {\left[\mathbf{A}_{1}\left(\mathbf{t}_{h}, \mathbf{u}_{h}\right),\left(\mathbf{s}_{h}, \mathbf{v}_{h}\right)\right]+\left[\mathbf{B}_{1}\left(\mathbf{s}_{h}, \mathbf{v}_{h}\right), \boldsymbol{\sigma}_{h}\right]+\left[\mathbf{B}^{p}\left(\mathbf{s}_{h}, \mathbf{v}_{h}\right), p_{h}\right]=\left[\mathbf{F}_{1},\left(\mathbf{s}_{h}, \mathbf{v}_{h}\right)\right],} \\
& {\left[\mathbf{B}_{1}\left(\mathbf{t}_{h}, \mathbf{u}_{h}\right), \boldsymbol{\tau}_{h}\right]+\left[\mathbf{B}^{\xi}\left(\boldsymbol{\tau}_{h}\right), \xi_{h}\right]=\left[\mathbf{F}_{2}, \boldsymbol{\tau}_{h}\right],} \\
& {\left[\mathbf{B}^{p}\left(\mathbf{t}_{h}, \mathbf{u}_{h}\right), q_{h}\right]+\left[\mathbf{B}^{\xi}\left(\boldsymbol{\sigma}_{h}\right), \eta_{h}\right] \quad=0,}
\end{aligned}
$$

for all $\left(\left(\mathbf{s}_{h}, \mathbf{v}_{h}\right), \tau_{h},\left(q_{h}, \eta_{h}\right)\right) \in X_{1, h} \times M_{1, h} \times M_{h}$.

Our purpose is to define these finite element subspaces so that (3.1) becomes well posed. As suggested by the linear version of Theorem 3.2 in [11], it suffices to prove the ellipticity of $\mathbf{A}_{1}$ on $X_{1, h}$ and the discrete inf-sup conditions for $\mathbf{B}$ and $\mathbf{B}_{1}$. In order to establish these properties 
we show below that the same arguments of the continuous case can be applied again, which will yield to determine the appropriate finite element spaces for each unknown. To begin with, we realize that there is nothing else to prove for $\mathbf{A}_{1}$ since the ellipticity of this bilinear form is certainly valid on any subspace of $X_{1}$. Next, in order to extend the proof of the continuous inf-sup condition for $\mathbf{B}$ to the discrete case, we require that $(0,0, \eta \mathbf{I})$ and $\left(q_{h} \mathbf{I}, 0,0\right)$ belong to $X_{1, h}^{\mathbf{t}} \times X_{1, h}^{\mathbf{u}} \times M_{1, h}$ for any $\left(q_{h}, \eta\right) \in M_{h}^{p} \times \mathbb{R}$, that is

$$
\eta \mathbf{I} \in M_{1, h} \quad \forall \eta \in \mathbb{R} \quad \text { and } \quad q_{h} \mathbf{I} \in X_{1, h}^{\mathbf{t}} \quad \forall q_{h} \in M_{h}^{p} .
$$

On the other hand, it is easy to see that the discrete kernel of the bilinear form $\mathbf{B}$ is given by $\tilde{X}_{1, h}^{\mathbf{t}} \times X_{1, h}^{\mathbf{u}} \times \tilde{M}_{1, h}$, where $\tilde{X}_{1, h}^{\mathbf{t}}:=\left\{s_{h} \in X_{1, h}^{\mathbf{t}}: \quad \int_{\Omega} q_{h} \operatorname{tr}\left(s_{h}\right)=0 \quad \forall q_{h} \in M_{h}^{p}\right\}$ and $\tilde{M}_{1, h}:=\left\{\boldsymbol{\tau}_{h} \in M_{1, h}: \quad \int_{\Omega} \operatorname{tr}\left(\boldsymbol{\tau}_{h}\right)=0\right\}$.

We note here that $\tilde{M}_{1, h}$ is clearly a subspace of $\tilde{M}_{1}$ and hence the equivalence of $\left\|\boldsymbol{\tau}_{h}\right\|_{\left[L^{2}(\Omega)\right]^{2 \times 2}}$ and $\left\|\operatorname{dev}\left(\boldsymbol{\tau}_{h}\right)\right\|_{\left[L^{2}(\Omega)\right]^{2 \times 2}}$ also holds for each $\boldsymbol{\tau}_{h} \in \tilde{M}_{1, h}$. Thus, in order to extend now the proof of the continuous inf-sup condition for $\mathbf{B}_{1}$ to the discrete case, we need that

$$
\operatorname{div}\left(\boldsymbol{\tau}_{h}\right) \in X_{1, h}^{\mathbf{u}} \quad \text { and } \quad \operatorname{dev}\left(\boldsymbol{\tau}_{h}\right) \in \tilde{X}_{1, h}^{\mathbf{t}} \quad \forall \boldsymbol{\tau}_{h} \in \tilde{M}_{1, h} .
$$

Since (3.2) and (3.3) do not impose any explicit condition on the elements of $M_{h}^{p}$, we choose this subspace of $L^{2}(\Omega)$ as the simplest possible one, that is, as the piecewise constant functions on the triangulation $\mathcal{T}_{h}$. Similarly, since the first restriction of (3.2) is satisfied if the piecewise constant tensors are included in $M_{1, h}$, we just choose this subspace of $H(\mathbf{d i v} ; \Omega)$ as the RaviartThomas space of order zero (see [5], [20]). Because of this choice of $M_{1, h}$, and in order to satisfy the first requirement of (3.3), we realize that it suffices to take $X_{1, h}^{\mathbf{u}}$ as the space of piecewise constant vectors on $\mathcal{T}_{h}$.

Finally, taking into account the choices already made for $M_{h}^{p}$ and $M_{1, h}$, and observing that the trace of any deviator is zero, we find that the remaining conditions in (3.2) and (3.3) are accomplished if $X_{1, h}^{\mathrm{t}}$ is chosen so that its restriction on each triangle $T \in \mathcal{T}_{h}$ becomes the local space $\mathcal{A}_{0}(T):=\langle\{\mathbf{I}\}\rangle \oplus \operatorname{dev}\left(\left[\mathcal{R} \mathcal{T}_{0}(T) \mathcal{R} \mathcal{T}_{0}(T)\right]^{\mathrm{t}}\right)$, where \langle\rangle is used hereafter to denote spanning, and

$$
\mathcal{R} \mathcal{T}_{0}(T):=\left\langle\left\{\left(\begin{array}{l}
1 \\
0
\end{array}\right),\left(\begin{array}{l}
0 \\
1
\end{array}\right),\left(\begin{array}{l}
x_{1} \\
x_{2}
\end{array}\right)\right\}\right\rangle
$$

is the local Raviart-Thomas space of order zero. Moreover, it is not difficult to see that

$$
\mathcal{A}_{0}(T):=\left[\mathbf{P}_{0}(T)\right]^{2 \times 2} \oplus\left\langle\left\{\left(\begin{array}{cc}
x_{1} & 2 x_{2} \\
0 & -x_{1}
\end{array}\right),\left(\begin{array}{cc}
-x_{2} & 0 \\
2 x_{1} & x_{2}
\end{array}\right)\right\}\right\rangle,
$$

where $\mathbf{P}_{0}(T)$ denotes the space of constant functions defined on $T$.

According to the above analysis, our finite element subspaces are given by

$$
\begin{gathered}
X_{1, h}^{\mathbf{t}}:=\left\{\mathbf{s} \in\left[L^{2}(\Omega)\right]^{2 \times 2}:\left.\quad \mathbf{s}\right|_{T} \in \mathcal{A}_{0}(T) \quad \forall T \in \mathcal{T}_{h}\right\}, \\
X_{1, h}^{\mathbf{u}}:=\left\{\mathbf{v} \in\left[L^{2}(\Omega)\right]^{2}:\left.\quad \mathbf{v}\right|_{T} \in\left[\mathbf{P}_{0}(T)\right]^{2} \quad \forall T \in \mathcal{T}_{h}\right\}, \\
M_{1, h}:=\left\{\boldsymbol{\tau} \in H(\operatorname{div} ; \Omega):\left.\quad\left(\tau_{i 1} \tau_{i 2}\right)^{\mathrm{t}}\right|_{T} \in \mathcal{R} \mathcal{T}_{0}(T) \quad \forall i \in\{1,2\}, \quad \forall T \in \mathcal{T}_{h}\right\}
\end{gathered}
$$

and

$$
M_{h}^{p}:=\left\{q \in L^{2}(\Omega):\left.\quad q\right|_{T} \in \mathbf{P}_{0}(T) \quad \forall T \in \mathcal{T}_{h}\right\} .
$$

The well posedness of the discrete problem (3.1) and the corresponding quasi-optimal error estimate can then be established. 
Theorem 3.1 Problem (3.1) has a unique solution $\left(\left(\mathbf{t}_{h}, \mathbf{u}_{h}\right), \boldsymbol{\sigma}_{h},\left(p_{h}, \xi_{h}\right)\right) \in X_{1, h} \times M_{1, h} \times M_{h}$. Moreover, there exist a positive constant $\hat{C}(\alpha, \nu)=O\left(\frac{\alpha^{3}}{\nu}\right)$, independent of $h$, such that

$$
\begin{aligned}
& \left\|((\mathbf{t}, \mathbf{u}), \boldsymbol{\sigma},(p, \xi))-\left(\left(\mathbf{t}_{h}, \mathbf{u}_{h}\right), \boldsymbol{\sigma}_{h},\left(p_{h}, \xi_{h}\right)\right)\right\| \\
& \leq \hat{C}(\alpha, \nu) \inf _{\left(\left(\mathbf{s}_{h}, \mathbf{v}_{h}\right), \boldsymbol{\tau}_{h}, q_{h}\right) \in X_{1, h} \times M_{1, h} \times M_{h}^{p}}\left\|((\mathbf{t}, \mathbf{u}), \boldsymbol{\sigma}, p)-\left(\left(\mathbf{s}_{h}, \mathbf{v}_{h}\right), \boldsymbol{\tau}_{h}, q_{h}\right)\right\|
\end{aligned}
$$

where $((\mathbf{t}, \mathbf{u}), \boldsymbol{\sigma},(p, \xi))$ is the unique solution of the continuous problem (2.6).

Proof. We first remark, since we are dealing with a linear problem, that the Céa estimate (3.5) is equivalent to stability of the Galerkin scheme (3.1). Now, as shown by our previous analysis, the present finite element subspaces guarantee the ellipticity of $\mathbf{A}_{1}$ on $X_{1, h}$, with the same constant $\nu$, as well as the discrete inf-sup conditions for $\mathbf{B}$ and $\mathbf{B}_{1}$, with constants depending only on $\Omega$. Hence, using again that $\left\|\mathbf{A}_{1}\right\|=O(\alpha)$ and that both $\left\|\mathbf{B}_{1}\right\|$ and $\|\mathbf{B}\|$ are of $O(1)$, we can apply the linear version of Theorem 3.2 in [11] to deduce the unique solvability of (3.1) and the corresponding stability with a constant behaving like $O\left(\frac{\alpha^{3}}{\nu}\right)$.

Next, we have the following result on the rate of convergence of the solution of (3.1).

TheOREM 3.2 Let $((\mathbf{t}, \mathbf{u}), \boldsymbol{\sigma},(p, \xi))$ and $\left(\left(\mathbf{t}_{h}, \mathbf{u}_{h}\right), \boldsymbol{\sigma}_{h},\left(p_{h}, \xi_{h}\right)\right)$ be the unique solutions of the continuous and discrete formulations, respectively. Assume that $\mathbf{t} \in\left[H^{1}(\Omega)\right]^{2 \times 2}, \mathbf{u} \in\left[H^{1}(\Omega)\right]^{2}$, $\boldsymbol{\sigma} \in\left[H^{1}(\Omega)\right]^{2 \times 2}, \operatorname{div}(\boldsymbol{\sigma}) \in\left[H^{1}(\Omega)\right]^{2}$, and $p \in H^{1}(\Omega)$. Then there exists a positive constant $\bar{C}(\alpha, \nu)=O\left(\frac{\alpha^{3}}{\nu}\right)$, independent of $h$, such that

$$
\begin{aligned}
& \left\|((\mathbf{t}, \mathbf{u}), \boldsymbol{\sigma},(p, \xi))-\left(\left(\mathbf{t}_{h}, \mathbf{u}_{h}\right), \boldsymbol{\sigma}_{h},\left(p_{h}, \xi_{h}\right)\right)\right\|_{X_{1} \times M_{1} \times M} \\
& \leq \bar{C}(\alpha, \nu) h\left(\|\mathbf{t}\|_{\left[H^{1}(\Omega)\right]^{2 \times 2}}+\|\boldsymbol{\sigma}\|_{\left[H^{1}(\Omega)\right]^{2 \times 2}}+\|\mathbf{u}\|_{\left[H^{1}(\Omega)\right]^{2}}+\|\operatorname{div}(\boldsymbol{\sigma})\|_{\left[H^{1}(\Omega)\right]^{2}}+\|p\|_{H^{1}(\Omega)}\right) .
\end{aligned}
$$

Proof. It is a consequence of the Céa estimate (3.5) and the well known approximation properties of the subspaces $X_{1, h}^{\mathbf{t}}, X_{1, h}^{\mathbf{u}}, M_{1, h}$ and $M_{h}^{p}$, which follow from classical error estimates for projection and equilibrium interpolation operators (see, e.g. [20]).

\section{A-posteriori error analysis}

We now develop an a-posteriori error analysis (based on suitable local problems) and derive reliable estimates for the mixed finite element solution introduced in the previous section. Similarly as in [13], our approach follows the technique from [6], which is a modification of the original Bank-Weiser method proposed in [3].

Let us first introduce some notations. We denote by $E_{h}$ the set of all the edges of the triangulation $\mathcal{T}_{h}$, define $E_{h}(\Gamma):=\left\{e \in E_{h}: \quad e \subseteq \Gamma\right\}$, and given $T \in \mathcal{T}_{h}$, we let $E(T):=\{e \in$ $\left.E_{h}: \quad e \subseteq \partial T\right\}$. In addition, the inner product of $H(\operatorname{div} ; T)$ is denoted by $\langle\cdot, \cdot\rangle_{H(\operatorname{div} ; T)}$, and $\mathbf{n}_{T}$ stands for the unit outward normal to $\partial T$.

On the other hand, given a polygonal domain $\mathcal{S} \subseteq \mathbb{R}^{2}$ and $m \in(1, \infty)$, the Sobolev space $W^{1, m}(\mathcal{S})$ is the Banach space of functions $v \in L^{m}(\mathcal{S})$ such that the first order distributional derivatives of $v$ are functions of $L^{m}(\mathcal{S})$. A Sobolev imbedding theorem establishes that $W^{1, m}(\mathcal{S}) \subseteq C(\overline{\mathcal{S}})$ if $m>2$ (see [18] for details). Also, it is well known that the trace theorem ensures the existence of a linear continuous map $\gamma: W^{1, m}(\mathcal{S}) \rightarrow L^{m}(\partial \mathcal{S})$ such that $\gamma v=\left.v\right|_{\partial \mathcal{S}}$ for each $v \in W^{1, m}(\mathcal{S}) \cap C(\overline{\mathcal{S}})$. The range of $\gamma$, which is a strict subspace of $L^{m}(\partial \mathcal{S})$, is denoted 
by $W^{1-1 / m, m}(\partial \mathcal{S})$. In particular, when $\mathcal{S}:=T \in \mathcal{T}_{h}$ and $m=2$, we use the standard notation and write $H^{1 / 2}(\partial T)$ instead of $W^{1 / 2,2}(\partial T)$.

Now, given an edge $e \in E(T), H_{0}^{1}(e)$ stands for the closure in $H^{1}(e)$ of the space of infinitely differentiable functions with compact support in $e$. We recall here that the interpolation space with index $1 / 2$ between $H_{0}^{1}(e)$ and $L^{2}(e)$ is $H_{00}^{1 / 2}(e)$ (cf. [18]). The space $H_{00}^{1 / 2}(e)$ may be alternatively defined as the subspace of functions in $H^{1 / 2}(e)$ whose extensions by zero to the rest of $\partial T$ belong to $H^{1 / 2}(\partial T)$. We will also need the dual space of $H^{1 / 2}(\partial T)$, which is denoted by $H^{-1 / 2}(\partial T)$. To this respect, we remark that the restriction of an element in $H^{-1 / 2}(\partial T)$ over $e$ does not belong in general to $H^{-1 / 2}(e)$, but to $H_{00}^{-1 / 2}(e)$, dual space of $H_{00}^{1 / 2}(e)$ pivotal to $L^{2}(e)$, and which is, therefore, larger than $H^{-1 / 2}(e)$. According to this, in what follows we set $\langle\cdot, \cdot\rangle_{e}$ for the duality pairing between $\left[H_{00}^{-1 / 2}(e)\right]^{2}$ and $\left[H_{00}^{1 / 2}(e)\right]^{2}$ with $\left[L^{2}(e)\right]^{2}$ as pivot space, and we let $\langle\cdot, \cdot\rangle_{\partial T}$ be the duality pairing between $\left[H^{-1 / 2}(\partial T)\right]^{2}$ and $\left[H^{1 / 2}(\partial T)\right]^{2}$ with $\left[L^{2}(\partial T)\right]^{2}$ as pivot space.

We now define the Riesz projection of the error with respect to the inner product of $X:=$ $X_{1} \times M_{1}$ as the unique element $(\overline{\mathbf{t}}, \overline{\mathbf{u}}, \overline{\boldsymbol{\sigma}}) \in X$ such that

$$
\langle(\overline{\mathbf{t}}, \overline{\mathbf{u}}, \overline{\boldsymbol{\sigma}}),(\mathbf{s}, \mathbf{v}, \boldsymbol{\tau})\rangle_{X}=\left[\mathbf{A}\left(\mathbf{t}-\mathbf{t}_{h}, \mathbf{u}-\mathbf{u}_{h}, \boldsymbol{\sigma}-\boldsymbol{\sigma}_{h}\right),(\mathbf{s}, \mathbf{v}, \boldsymbol{\tau})\right]+\left[\mathbf{B}(\mathbf{s}, \mathbf{v}, \boldsymbol{\tau}),\left(p-p_{h}, \xi-\xi_{h}\right)\right]
$$

for all $(\mathbf{s}, \mathbf{v}, \boldsymbol{\tau}) \in X$, where $\mathbf{A}$ and $\mathbf{B}$ are the bilinear forms defined in Section 2, and

$$
\langle(\overline{\mathbf{t}}, \overline{\mathbf{u}}, \overline{\boldsymbol{\sigma}}),(\mathbf{s}, \mathbf{v}, \boldsymbol{\tau})\rangle_{X}:=\langle\overline{\mathbf{t}}, \mathbf{s}\rangle_{\left[L^{2}(\Omega)\right]^{2 \times 2}}+\langle\overline{\mathbf{u}}, \mathbf{v}\rangle_{\left[L^{2}(\Omega)\right]^{2}}+\langle\overline{\boldsymbol{\sigma}}, \boldsymbol{\tau}\rangle_{H(\operatorname{div} ; \Omega)} .
$$

In what follows, we assume that there exists $m>2$ such that the Dirichlet data $\mathbf{g} \in$ $\left[H^{1 / 2}(\Gamma) \cap W^{1-1 / m, m}(\Gamma)\right]^{2}$ and let $\varphi_{h}$ be a given auxiliary function in $\left[H^{1}(\Omega) \cap W^{1, m}(\Omega)\right]^{2}$ such that $\varphi_{h}(\overline{\mathbf{x}})=\mathbf{g}(\overline{\mathbf{x}})$ for each vertex $\overline{\mathbf{x}}$ of $\mathcal{T}_{h}$ lying on $\Gamma$. In addition, for each $T \in \mathcal{T}_{h}$ we let $\hat{\boldsymbol{\sigma}}_{T} \in H(\operatorname{div} ; T)$ be the unique solution of the local problem

$$
\left\langle\hat{\boldsymbol{\sigma}}_{T}, \boldsymbol{\tau}\right\rangle_{H(\operatorname{div} ; T)}=\mathcal{F}_{h, T}(\boldsymbol{\tau}) \quad \forall \boldsymbol{\tau} \in H(\operatorname{div} ; T),
$$

where $\mathcal{F}_{h, T} \in H(\operatorname{div} ; T)^{\prime}$ is defined by

$$
\begin{aligned}
\mathcal{F}_{h, T}(\boldsymbol{\tau}) & :=\int_{T} \boldsymbol{\tau}: \mathbf{t}_{h}+\int_{T} \mathbf{u}_{h} \cdot \operatorname{div}(\boldsymbol{\tau})-\xi_{h} \int_{T} \operatorname{tr}(\boldsymbol{\tau}) \\
& -\left\langle\boldsymbol{\tau} \mathbf{n}_{T}, \varphi_{h}\right\rangle_{\partial T}+\sum_{e \in E(T) \cap E_{h}(\Gamma)}\left\langle\boldsymbol{\tau} \mathbf{n}_{T}, \varphi_{h}-\mathbf{g}\right\rangle_{e} .
\end{aligned}
$$

The following lemma provides an upper bound for $\|(\overline{\mathbf{t}}, \overline{\mathbf{u}}, \overline{\boldsymbol{\sigma}})\|_{X}$.

LEMMA 4.1 There holds

$$
\|(\overline{\mathbf{t}}, \overline{\mathbf{u}}, \overline{\boldsymbol{\sigma}})\|_{X}^{2} \leq \sum_{T \in \mathcal{T}_{h}} \hat{\theta}_{T}^{2}
$$

where

$$
\hat{\theta}_{T}^{2}:=\left\|\hat{\boldsymbol{\sigma}}_{T}\right\|_{H(\operatorname{div} ; T)}^{2}+\left\|\boldsymbol{\sigma}_{h}-\nu \mathbf{t}_{h}+p_{h} \mathbf{I}\right\|_{\left[L^{2}(T)\right]^{2 \times 2}}^{2}+\left\|\mathbf{f}+\operatorname{div}\left(\boldsymbol{\sigma}_{h}\right)-\alpha \mathbf{u}_{h}\right\|_{\left[L^{2}(T)\right]^{2}}^{2} .
$$

Proof. The proof utilizes the same arguments of Lemma 3.1 in [13]. We only remark here that the regularity hypotheses on $\mathbf{g}$ and $\varphi_{h}$ guarantee, by virtue of a Sobolev imbedding theorem, that $\mathbf{g}$ and $\varphi_{h}$ are both continuous, and hence the associated interpolation condition for the vertices of $\mathcal{T}_{h}$ lying on $\Gamma$ makes sense. We omit further details.

A priori estimates for the solutions of the local problems (4.1) are given in the following lemma. 
Lemma 4.2 There exists $C>0$, independent of $h, \alpha, \nu$, and $T$, such that

$$
\begin{gathered}
\left\|\hat{\boldsymbol{\sigma}}_{T}\right\|_{H(\mathbf{d i v} ; T)}^{2} \leq C\left(\left\|\mathbf{t}_{h}-\nabla \varphi_{h}\right\|_{\left[L^{2}(T)\right]^{2 \times 2}}^{2}+\left\|\mathbf{u}_{h}-\varphi_{h}\right\|_{\left[L^{2}(T)\right]^{2}}^{2}\right. \\
\left.+h_{T}^{2}\left|\xi_{h}\right|^{2}+\sum_{e \in E(T) \cap E_{h}(\Gamma)}\left\|\varphi_{h}-\mathbf{g}\right\|_{\left[H_{00}^{1 / 2}(e)\right]^{2}}^{2}\right) .
\end{gathered}
$$

Furthermore, for any $\mathbf{z} \in\left[H^{1}(\Omega) \cap W^{1, m}(\Omega)\right]^{2}$, with $m>2$, such that $\mathbf{z}=\mathbf{g}$ on $\Gamma$, we get

$$
\begin{aligned}
&\left\|\hat{\boldsymbol{\sigma}}_{T}\right\|_{H(\operatorname{div} ; T)}^{2} \leq C\left(\left\|\mathbf{t}_{h}-\nabla \mathbf{z}\right\|_{\left[L^{2}(T)\right]^{2 \times 2}}^{2}+\left\|\mathbf{u}_{h}-\mathbf{z}\right\|_{\left[L^{2}(T)\right]^{2}}^{2}+h_{T}^{2}\left|\xi_{h}\right|^{2}+\left\|\mathbf{J}_{h, T}(\mathbf{z})\right\|_{\left[H^{1 / 2}(\partial T)\right]^{2}}^{2}\right), \\
& \text { where } \mathbf{J}_{h, T}(\mathbf{z}):=\left\{\begin{array}{ll}
0 & \text { on } \partial T \cap \Gamma \\
\mathbf{z}-\varphi_{h} & \text { otherwise }
\end{array} .\right.
\end{aligned}
$$

Proof. It is clear from (4.1) that $\left\|\hat{\boldsymbol{\sigma}}_{T}\right\|_{H(\operatorname{div} ; T)}=\left\|\mathcal{F}_{h, T}\right\|_{H(\mathbf{d i v} ; T)^{\prime}}$. Therefore, the first estimate for $\left\|\hat{\boldsymbol{\sigma}}_{T}\right\|_{H(\mathbf{d i v} ; T)}$ follows after applying Gauss' formula to the expression $\left\langle\boldsymbol{\tau} \mathbf{n}_{T}, \varphi_{h}\right\rangle_{\partial T}$ appearing in the definition of the functional $\mathcal{F}_{h, T}$, and reordering the resulting terms so that the CauchySchwarz inequality can be applied conveniently. The proof of the second estimate is similar. One just needs to observe, after simple computations, that

$$
-\left\langle\boldsymbol{\tau} \mathbf{n}_{T}, \varphi_{h}\right\rangle_{\partial T}+\sum_{e \in E(T) \cap E_{h}(\Gamma)}\left\langle\boldsymbol{\tau} \mathbf{n}_{T}, \varphi_{h}-\mathbf{g}\right\rangle_{e}=-\left\langle\boldsymbol{\tau} \mathbf{n}_{T}, \mathbf{z}\right\rangle_{\partial T}+\left\langle\boldsymbol{\tau} \mathbf{n}_{T}, \mathbf{J}_{h, T}(\mathbf{z})\right\rangle .
$$

The rest proceeds as in the first case, applying now Gauss' formula to $\left\langle\boldsymbol{\tau} \mathbf{n}_{T}, \mathbf{z}\right\rangle_{\partial T}$.

The above lemmata allow us to establish next the main a-posteriori error estimates.

THEOREM 4.1 There exists a positive constant $C_{0}(\alpha, \nu)=O\left(\frac{\alpha^{3}}{\nu}\right)$, independent of $h$, such that

$$
\left\|(\mathbf{t}, \mathbf{u}, \boldsymbol{\sigma}, p, \xi)-\left(\mathbf{t}_{h}, \mathbf{u}_{h}, \boldsymbol{\sigma}_{h}, p_{h}, \xi_{h}\right)\right\|_{X_{1} \times M_{1} \times M} \leq C_{0}(\alpha, \nu)\left\{\sum_{T \in \mathcal{T}_{h}} \tilde{\theta}_{T}^{2}\right\}^{1 / 2}
$$

and

$$
\left\|(\mathbf{t}, \mathbf{u}, \boldsymbol{\sigma}, p, \xi)-\left(\mathbf{t}_{h}, \mathbf{u}_{h}, \boldsymbol{\sigma}_{h}, p_{h}, \xi_{h}\right)\right\|_{X_{1} \times M_{1} \times M} \leq C_{0}(\alpha, \nu)\left\{\sum_{T \in \mathcal{T}_{h}} \theta_{T}^{2}\right\}^{1 / 2},
$$

where for each triangle $T \in \mathcal{T}_{h}$, we define

$$
\begin{aligned}
& \tilde{\theta}_{T}^{2}:=\left\|\hat{\boldsymbol{\sigma}}_{T}\right\|_{H(\operatorname{div} ; T)}^{2}+\left\|\boldsymbol{\sigma}_{h}-\nu \mathbf{t}_{h}+p_{h} \mathbf{I}\right\|_{\left[L^{2}(T)\right]^{2 \times 2}}^{2} \\
& +\left\|\mathbf{f}+\operatorname{div}\left(\boldsymbol{\sigma}_{h}\right)-\alpha \mathbf{u}_{h}\right\|_{\left[L^{2}(T)\right]^{2}}^{2}+\left\|\operatorname{tr}\left(\mathbf{t}_{h}\right)\right\|_{L^{2}(T)}^{2},
\end{aligned}
$$

and

$$
\begin{aligned}
& \theta_{T}^{2}:=\left\|\mathbf{t}_{h}-\nabla \varphi_{h}\right\|_{\left[L^{2}(T)\right]^{2 \times 2}}^{2}+\left\|\mathbf{u}_{h}-\varphi_{h}\right\|_{\left[L^{2}(T)\right]^{2}}^{2}+h_{T}^{2}\left|\xi_{h}\right|^{2} \\
& +\sum_{e \in E(T) \cap E_{h}(\Gamma)}\left\|\varphi_{h}-\mathbf{g}\right\|_{\left[H_{00}^{1 / 2}(e)\right]^{2}}^{2}+\left\|\boldsymbol{\sigma}_{h}-\nu \mathbf{t}_{h}+p_{h} \mathbf{I}\right\|_{\left[L^{2}(T)\right]^{2 \times 2}}^{2} \\
& \quad+\left\|\mathbf{f}+\operatorname{div}\left(\boldsymbol{\sigma}_{h}\right)-\alpha \mathbf{u}_{h}\right\|_{\left[L^{2}(T)\right]^{2}}^{2}+\left\|\operatorname{tr}\left(\mathbf{t}_{h}\right)\right\|_{L^{2}(T)}^{2} .
\end{aligned}
$$


Proof. It is similar to the proof of Theorem 2.1 in [13]. Indeed, the continuous dependence result given in Theorem 2.1 (cf. (2.9)) is equivalent to the global inf-sup condition for the linear operator obtained by adding the three equations of the left hand side of (2.6). Hence, by applying this condition to the error $(\mathbf{t}, \mathbf{u}, \boldsymbol{\sigma}, p, \xi)-\left(\mathbf{t}_{h}, \mathbf{u}_{h}, \boldsymbol{\sigma}_{h}, p_{h}, \xi_{h}\right)$, and using the definition of the Riesz projection $(\overline{\mathbf{t}}, \overline{\mathbf{u}}, \overline{\boldsymbol{\sigma}}) \in X$, the definition of the operator $\mathbf{B}$, the third equation of the continuous problem (2.6), and Lemmas 4.1 and 4.2, we obtain the estimates (4.3) and (4.4). The details are omitted.

According to the previous theorem, we now introduce the reliable a-posteriori error estimates

$$
\tilde{\boldsymbol{\theta}}:=\left\{\sum_{T \in \mathcal{T}_{h}} \tilde{\theta}_{T}^{2}\right\}^{1 / 2} \quad \text { and } \quad \boldsymbol{\theta}:=\left\{\sum_{T \in \mathcal{T}_{h}} \theta_{T}^{2}\right\}^{1 / 2} .
$$

In addition, we establish next that $\tilde{\boldsymbol{\theta}}$ is quasi-efficient, which means that it is efficient up to a term depending on the traces $\left(\mathbf{u}-\varphi_{h}\right)$ on the edges of $\mathcal{T}_{h}$. We also remark that, besides the regularity and interpolation conditions, Lemma 4.1, Lemma 4.2, and Theorem 4.1 do not require any further assumptions on $\varphi_{h}$. However, as we show below, the above mentioned quasi-efficiency will restrict the possible choices of this auxiliary function.

Lemma 4.3 Assume that $\mathbf{u} \in\left[W^{1, m}(\Omega)\right]^{2}$, with $m>2$. Then there exists a positive constant $C_{1}(\alpha)=O\left(\alpha^{2}\right)$, independent of $h$, such that for all $T \in \mathcal{T}_{h}$

$$
\begin{aligned}
\tilde{\theta}_{T}^{2} \leq & C_{1}(\alpha)\left\{\left\|\mathbf{t}-\mathbf{t}_{h}\right\|_{\left[L^{2}(T)\right]^{2 \times 2}}^{2}+\left\|\mathbf{u}-\mathbf{u}_{h}\right\|_{\left[L^{2}(T)\right]^{2}}^{2}+\left\|\boldsymbol{\sigma}-\boldsymbol{\sigma}_{h}\right\|_{H(\operatorname{div} ; T)}^{2}\right. \\
& \left.+\left\|p-p_{h}\right\|_{L^{2}(T)}^{2}+h_{T}^{2}\left|\xi-\xi_{h}\right|^{2}+\left\|\mathbf{J}_{h, T}(\mathbf{u})\right\|_{\left[H^{1 / 2}(\partial T)\right]^{2}}^{2}\right\}
\end{aligned}
$$

and hence

$$
\begin{aligned}
\tilde{\boldsymbol{\theta}}^{2} \leq C_{1}(\alpha)\{ & \left\|(\mathbf{t}, \mathbf{u}, \boldsymbol{\sigma}, p, \xi)-\left(\mathbf{t}_{h}, \mathbf{u}_{h}, \boldsymbol{\sigma}_{h}, p_{h}, \xi_{h}\right)\right\|_{X_{1} \times M_{1} \times M}^{2} \\
& \left.+\sum_{T \in \mathcal{T}_{h}}\left\|\mathbf{J}_{h, T}(\mathbf{u})\right\|_{\left[H^{1 / 2}(\partial T)\right]^{2}}^{2}\right\},
\end{aligned}
$$

where $\mathbf{J}_{h, T}(\mathbf{u}):=\left\{\begin{array}{ll}0 & \text { on } \partial T \cap \Gamma \\ \mathbf{u}-\varphi_{h} & \text { otherwise }\end{array}\right.$.

Proof. It is similar to the proof of Lemma 4.1 in [13]. We omit the details here.

At this point we remark that, although the reliable a-posteriori error estimate $\tilde{\boldsymbol{\theta}}$ is also quasi-efficient, its eventual applicability is limited by the fact that it requires the knowledge of the exact solutions $\hat{\boldsymbol{\sigma}}_{T}$ of the local problems (4.1), which live all in the infinite dimensional space $H(\operatorname{div} ; T)$. This difficulty can be partially overcomed by using $h, p$ or $h-p$ versions of the finite element method to solve (4.1) approximately, which naturally yields approximations of the local indicators $\tilde{\theta}_{T}$, and hence of $\tilde{\boldsymbol{\theta}}$. We will go back to this point in Section 5 .

On the other hand, the advantage of $\boldsymbol{\theta}$, which is not necessarily quasi-efficient, is that it does not need the exact or any approximate solution of (4.1), but a suitable $\varphi_{h}$. In order to choose this auxiliary function, we adopt the criterion of enforcing the quasi-efficiency of $\tilde{\boldsymbol{\theta}}$ so that it becomes closer to full efficiency. As established in Lemma 4.3, this criterion requires that the traces $\left.\varphi_{h}\right|_{\partial T}$ be as close as possible to the exact traces $\left.\mathbf{u}\right|_{\partial T}$, for each $T \in \mathcal{T}_{h}$. According to this, and taking into account the finite element subspaces defining the discrete scheme, we propose 
next a heuristic procedure to choose $\varphi_{h}$. Hereafter, given a nonnegative integer $l, \mathbf{P}_{l}(T)$ denotes the space of polynomials of degree $\leq l$ defined on $T$.

We first compute local functions $\varphi_{h, T}$, for each $T \in \mathcal{T}_{h}$, satisfying:

1. $\varphi_{h, T} \in\left[\mathbf{P}_{2}(T)\right]^{2}$.

2. $\nabla \varphi_{h, T}$ is the $\left[L^{2}(T)\right]^{2 \times 2}$ - projection of $\left.\mathbf{t}_{h}\right|_{T}$ onto the space $\nabla\left[\mathbf{P}_{2}(T)\right]^{2}$.

3. $\varphi_{h, T}\left(\overline{\mathbf{x}}_{T}\right)=\left.\mathbf{u}_{h}\right|_{T}$, where $\overline{\mathbf{x}}_{T}$ is the barycenter of $T$.

It is easy to see that each $\varphi_{h, T}$ is uniquely determined by the above conditions. Then, similarly as in [15], we now define $\varphi_{h}$ as the continuous average of the functions $\varphi_{h, T}$. In other words, $\varphi_{h}$ is the unique function in $[C(\bar{\Omega})]^{2}$ satisfying:

1. $\left.\varphi_{h}\right|_{T} \in\left[\mathbf{P}_{2}(T)\right]^{2}$ for each $T \in \mathcal{T}_{h}$.

2. For each vertex $\overline{\mathbf{x}}$ of $\mathcal{T}_{h}$ lying on $\Gamma$ and for each middle point $\overline{\mathbf{x}}$ of the edges $e \in E_{h}(\Gamma)$, $\varphi_{h}(\overline{\mathbf{x}})=\mathbf{g}(\overline{\mathbf{x}})$.

3. For each vertex $\overline{\mathbf{x}}$ of $\mathcal{T}_{h}$ lying in $\Omega$ and for each middle point $\overline{\mathbf{x}}$ of the edges $e \in E_{h}-E_{h}(\Gamma)$, $\varphi_{h}(\overline{\mathbf{x}})$ is the average of the values $\varphi_{h, T}(\overline{\mathbf{x}})$ on all the triangles $T \in \mathcal{T}_{h}$ to which $\overline{\mathbf{x}}$ belongs.

We observe here that the computations of the local functions $\varphi_{h, T}$ (through a projection argument) and the global $P_{2}$-interpolant $\varphi_{h}$ are standard procedures in the finite element method. Moreover, they are comparable (in complexity) to the computations of approximate solutions of the local problems (4.1) via higher order $H($ div; $T$ ) subspaces.

\section{Numerical results}

In this section we provide several numerical examples illustrating the performance of the mixed finite element scheme (3.1) and the fully explicit a-posteriori error estimate $\boldsymbol{\theta}$ (cf. (4.6), (4.7)) with the above described choice of the auxiliary function $\varphi_{h}$.

Hereafter, $N$ is the number of degrees of freedom defining the subspaces $X_{1, h}, M_{1, h}$, and $M_{h}$, that is, $N:=9$ (number of triangles of $\left.\mathcal{T}_{h}\right)+2$ (number of edges of $\mathcal{T}_{h}$ ) +1 , which leads asymptotically to 12 unknowns per triangle. In order to compare this amount with the number of unknowns employed by more traditional approaches, we consider the $\mathbf{P}_{1}$-iso $\mathbf{P}_{1}$ finite element pair for the usual primal-mixed variational formulation of the generalized Stokes problem. In this case, the pressure is approximated by continuous piecewise linear elements on a triangular mesh, and the velocity is approximated by continuous piecewise linear elements on the finer mesh obtained by refining each element in the pressure mesh into four elements using the midpoints of each side. It is not difficult to see that these finite element subspaces lead asymptotically to 7.5 degrees of freedom per triangle. We emphasize then that the additional cost of 4.5 unknowns per triangle in our present mixed approach is certainly justified by the fact that two other quantities of physical interest $(\mathbf{t}$ and $\boldsymbol{\sigma}$ ) are approximated directly, without any need of numerical postprocessing. In addition, on the contrary to the $\mathbf{P}_{1}$-iso $\mathbf{P}_{1}$ approximation where one has to impose continuity requirements at each node for the pressure $p_{h}$ and at each node and midpoint for the components of the velocity $\mathbf{u}_{h}$, we only need here to consider the continuity of the normal components of the flux $\boldsymbol{\sigma}_{h}$ at the edges of the triangulation. This yields therefore a much simpler computation of the global stiffness matrix of the present mixed finite element 
method since all the sub-matrices connecting only the other three main unknowns $\left(\mathbf{t}_{h}, \mathbf{u}_{h}\right.$, and $p_{h}$ ) become block-diagonal (in a general sense including rectangular matrices). Consequently, computing in particular the inverse of the sub-matrix generated by $\mathbf{t}_{h}$ and $\mathbf{u}_{h}$ is straightforward, which allows to eliminate these unknowns, thus simplifying significantly the solution procedure of the whole discrete system (3.1).

We now provide further notations. The individual and global errors are defined as follows

$$
\begin{gathered}
\mathbf{e}(\mathbf{t}):=\left\|\mathbf{t}-\mathbf{t}_{h}\right\|_{\left[L^{2}(\Omega)\right]^{2 \times 2}}, \quad \mathbf{e}(\mathbf{u}):=\left\|\mathbf{u}-\mathbf{u}_{h}\right\|_{\left[L^{2}(\Omega)\right]^{2}}, \\
\mathbf{e}(\boldsymbol{\sigma}):=\left\|\boldsymbol{\sigma}-\boldsymbol{\sigma}_{h}\right\|_{H(\operatorname{div} ; \Omega)}, \quad \mathbf{e}(p):=\left\|p-p_{h}\right\|_{L^{2}(\Omega)}, \quad \mathbf{e}(\xi):=\left|\xi-\xi_{h}\right|,
\end{gathered}
$$

and

$$
\mathbf{e}:=\left\{[\mathbf{e}(\mathbf{t})]^{2}+[\mathbf{e}(\boldsymbol{\sigma})]^{2}+[\mathbf{e}(p)]^{2}+[\mathbf{e}(\mathbf{u})]^{2}+[\mathbf{e}(\xi)]^{2}\right\}^{1 / 2},
$$

where $(\mathbf{t}, \mathbf{u}, \boldsymbol{\sigma}, p, \xi)$ and $\left(\mathbf{t}_{h}, \mathbf{u}_{h}, \boldsymbol{\sigma}_{h}, p_{h}, \xi_{h}\right)$ are the unique solutions of the continuous and discrete mixed formulations, respectively. In addition, the effectivity index associated to the a-posteriori error estimate $\boldsymbol{\theta}$ is given by $\mathbf{e} / \boldsymbol{\theta}$. Also, given two consecutive triangulations with degrees of freedom $N$ and $N^{\prime}$, and corresponding total errors given by $\mathbf{e}$ and $\mathbf{e}^{\prime}$, the experimental rate of convergence is defined by $\gamma:=-2 \frac{\log \left(\mathbf{e} / \mathbf{e}^{\prime}\right)}{\log \left(N / N^{\prime}\right)}$.

On the other hand, the adaptive algorithm used in the mesh refinement process is the following (see [23]):

1. Start with a coarse mesh $\mathcal{T}_{h}$.

2. Solve the discrete problem (3.1) for the current mesh $\mathcal{T}_{h}$.

3. Compute the auxiliary function $\varphi_{h}$.

4. Compute $\theta_{T}$ for each triangle $T \in \mathcal{T}_{h}$.

5. Evaluate stopping criterion and decide to finish or go to next step.

6. Use blue-green procedure to refine each $T^{\prime} \in \mathcal{T}_{h}$ whose indicator $\theta_{T^{\prime}}$ satisfies

$$
\theta_{T^{\prime}} \geq \frac{1}{2} \max \left\{\theta_{T}: \quad T \in \mathcal{T}_{h}\right\} .
$$

7. Define resulting mesh as actual mesh $\mathcal{T}_{h}$ and go to step 2.

We remark here that the $H^{1 / 2}$-norm appearing in the computation of $\theta_{T}$ (cf. (4.6)) is approximated by means of an interpolation estimate, that is, given $e \in E(T) \cap E_{h}(\Gamma)$, we consider the bound

$$
\left\|\varphi_{h}-\mathbf{g}\right\|_{\left[H_{00}^{1 / 2}(e)\right]^{2}}^{2} \leq\left\|\varphi_{h}-\mathbf{g}\right\|_{\left[L^{2}(e)\right]^{2}}\left\|\varphi_{h}-\mathbf{g}\right\|_{\left[H_{0}^{1}(e)\right]^{2}} .
$$

The numerical results presented below were obtained in a Compaq Alpha ES40 Parallel Computer using a MATLAB code. We consider four examples of the generalized Stokes problem (1.1), with different choices of the parameters $\alpha$ and $\nu$ for three of them. The data $\mathbf{f}$ and $\mathbf{g}$ are chosen so that the velocity $\mathbf{u}:=\left(u_{1}, u_{2}\right)^{\mathrm{t}}$ and the pressure $p$ are the ones shown below in Table 5.0. Example 1 considers smooth solutions for both unknowns. Then, Examples 2 and 
4 illustrate the case of velocities with boundary and inner layers, respectively. More precisely, $\mathbf{u}$ has a boundary layer around the origin in Example 2, while $\mathbf{u}$ has an inner layer around the line $x_{2}=0.5-x_{1}$ in Example 4. Finally, Example 3 considers the case of $\mathbf{u}$ with a singularity around the exterior neighborhood of the boundary point $(1,1)$. Certainly, the velocities of the four examples are divergence free.

Table 5.0: Domain and exact solution for each example.

\begin{tabular}{|c|c|c|c|c|}
\hline EXAMPLE & $\Omega$ & $u_{1}\left(x_{1}, x_{2}\right)$ & $u_{2}\left(x_{1}, x_{2}\right)$ & $p\left(x_{1}, x_{2}\right)$ \\
\hline 1 & $(-1,1)^{2}$ & $-e^{x_{1}}\left(x_{2} \cos \left(x_{2}\right)+\sin \left(x_{2}\right)\right)$ & $e^{x_{1}} x_{2} \sin \left(x_{2}\right)$ & $2 e^{x_{1}} \sin \left(x_{2}\right)$ \\
\hline 2 & $(0,1)^{2}$ & $-\sqrt{\alpha} e^{-\sqrt{\alpha}\left(x_{1}+x_{2}\right)}$ & $-u_{1}\left(x_{1}, x_{2}\right)$ & $2 e^{2 x_{1}-1} \sin \left(2 x_{2}-1\right)$ \\
\hline 3 & $(-1,1)^{2}$ & $-\left(2.1-x_{1}-x_{2}\right)^{-1 / 3}$ & $-u_{1}\left(x_{1}, x_{2}\right)$ & $2 e^{x_{1}} \sin \left(x_{2}\right)$ \\
\hline 4 & $(0,1)^{2}$ & $-\sqrt{\alpha} e^{-\sqrt{\alpha}\left(0.5-x_{1}-x_{2}\right)^{2}}$ & $-u_{1}\left(x_{1}, x_{2}\right)$ & $2 e^{2 x_{1}-1} \sin \left(2 x_{2}-1\right)$ \\
\hline
\end{tabular}

Before commenting on the numerical results obtained, we observe in advance that the information on the individual error $\mathbf{e}(\xi)$ is not displayed below since it converges very rapidly to zero in all the examples considered.

In Tables 5.1 and 5.2 we give the individual and global errors, the effectivity index $\mathbf{e} / \boldsymbol{\theta}$, and the experimental rate of convergence $\gamma$ for the uniform refinement as applied to Example 1 with pairs of parameters $(\alpha, \nu)=(10,1)$ and $(\alpha, \nu)=(100,1)$. In addition, Figures 5.1.1 and 5.2.1 show the corresponding individual errors versus the degrees of freedom $N$. We observe here that the rates of convergence behave as predicted by the theory, that is, of $O(h)$, and that, due to the order of the constant $\bar{C}(\alpha, \nu)$ in Theorem 3.2, some of these rates begin to deteriorate as $\alpha$ increases. We also notice that the dominant components of the global error are given by $\mathbf{e}(\mathbf{t})$ and $\mathbf{e}(\boldsymbol{\sigma})$. Further, the effectivity indexes remain bounded above and below as $N$ increases (with smaller lower bounds for bigger $\alpha$ ), which confirms the reliability of $\boldsymbol{\theta}$ and provides numerical evidences for it being efficient. However, as shown by Figures 5.1.2 and 5.2.2, and due to the fact that the solution of Example 1 is smooth, there is no relevant difference between the uniform and adaptive procedures for the global error e versus $N$.

The numerical results concerning Example 2 are presented in Tables 5.3 and 5.4 where we display the individual and global errors, the effectivity index $\mathbf{e} / \boldsymbol{\theta}$, and the experimental rate of convergence $\gamma$ for both refinements with $(\alpha, \nu)=(100,0.5)$ and $(\alpha, \nu)=(1000,0.5)$. We note from these tables that only $\mathbf{e}(\boldsymbol{\sigma})$ constitutes now the dominant part of $\mathbf{e}$. In addition, we observe a very clear difference between the uniform and adaptive refinements. The global error $\mathbf{e}$ of the latter decreases much faster than that of the former, thus recovering the rate of convergence $O(h)$. As shown in Figures 5.3 and 5.4, this fact is even more pronounced for $\alpha=1000$ where the convergence of the uniform refinement is very slow. However, although the effectivity indexes remain bounded above and below as $N$ increases, for the two pairs of parameters and for both refinements, they also increase as $\alpha$ gets larger. Next, Meshes 5.3 and 5.4 display some intermediate meshes obtained with the adaptive refinement algorithm. It is interesting to confirm, as expected, that the procedure is able to recognize the boundary layer around $(0,0)$. Also, we remark that this refinement is even more localized near the origin for $\alpha=1000$, which is due to the fact that this layer becomes thinner as $\alpha$ gets larger. 
Next, Tables 5.5 and 5.6 provide the numerical results obtained for Example 3 with $(\alpha, \nu)=$ $(10,1)$ and $(\alpha, \nu)=(100,1)$. As for the previous examples, $\mathbf{e}(\boldsymbol{\sigma})$ is again the dominant part of the global error e. Also, we observe that the effectivity indexes remain bounded above and below as the number of degrees of freedom $N$ increases, with bounds close to 1.0, which confirms the reliability of $\boldsymbol{\theta}$ and constitutes numerical evidences of its eventual efficiency. In addition, according to the experimental rates of convergence, which are also illustrated by Figures 5.5 and 5.6, the adaptive procedure yields again the quasi-optimal rate of convergence $O(h)$ for the global error e. Moreover, as expected, the adaptive refinement algorithm is able to identify the singularities of the problem. In fact, as shown by Meshes 5.5 and 5.6, the adapted meshes are highly refined around the boundary point $(1,1)$, in whose outer neighborhood the singularity lives. Further, similarly as for Example 2, the refinement is even more localized as $\alpha$ gets larger.

Finally, the numerical results concerning Example 4 with $(\alpha, \nu)=(1000,0.5)$ are collected in Table 5.7. The remarks and conclusions here are similar to those for Examples 2 and 3. Again, the effectivity indexes remain bounded, with bounds around 0.11, and, although the experimental rates of convergence of both refinements aproach 1 as $N$ incresases, the global error of the adaptive one begins to decrease before than the uniform one. This fact is clearly observed in Figure 5.7 where the curve e versus $N$ is shown. In addition, as expected, the corresponding adaptive refinement algorithm is able to recognize the inner layer of the problem. Indeed, as can be seen in Meshes 5.7, the adapted meshes are highly refined around the line $x_{2}=0.5-x_{1}$. We also notice here that the refinements identify a thin band exactly on this line, which corresponds to the flat behaviour of the solution caused by the power 2 in the exponent of the exponential function.

On the other hand, although we mentioned before that the solutions of the local problems (4.1) could be approximated via higher order $H(\operatorname{div} ; T)$ subspaces, we show next that this additional computational effort would not necessarily improve the efficiency of the a-posteriori error estimate. To this end, we now identify the main components of $\boldsymbol{\theta}$, which are given by those terms providing, respectively, the a-priori bounds for the local solutions $\hat{\boldsymbol{\sigma}}_{T}$, and the residuals of the constitutive, equilibrium, and compressibility equations. More precisely, according to (4.6) and (4.7), we can write $\boldsymbol{\theta}=\left\{\boldsymbol{\theta}_{\varphi}^{2}+\boldsymbol{\theta}_{\text {res }}^{2}\right\}^{1 / 2}$, where

$\boldsymbol{\theta}_{\varphi}^{2}:=\sum_{T \in \mathcal{T}_{h}}\left\{\left\|\mathbf{t}_{h}-\nabla \varphi_{h}\right\|_{\left[L^{2}(T)\right]^{2 \times 2}}^{2}+\left\|\mathbf{u}_{h}-\varphi_{h}\right\|_{\left[L^{2}(T)\right]^{2}}^{2}+h_{T}^{2}\left|\xi_{h}\right|^{2}\right\}+\sum_{e \in E_{h}(\Gamma)}\left\|\varphi_{h}-\mathbf{g}\right\|_{\left[H_{00}^{1 / 2}(e)\right]^{2}}^{2}$,

and

$$
\boldsymbol{\theta}_{r e s}^{2}:=\sum_{T \in \mathcal{T}_{h}}\left\{\left\|\boldsymbol{\sigma}_{h}-\nu \mathbf{t}_{h}+p_{h} \mathbf{I}\right\|_{\left[L^{2}(T)\right]^{2 \times 2}}^{2}+\left\|\mathbf{f}+\operatorname{div}\left(\boldsymbol{\sigma}_{h}\right)-\alpha \mathbf{u}_{h}\right\|_{\left[L^{2}(T)\right]^{2}}^{2}+\left\|\operatorname{tr}\left(\mathbf{t}_{h}\right)\right\|_{L^{2}(T)}^{2}\right\} .
$$

Then, in Table 5.8 we display the components $\boldsymbol{\theta}_{\varphi}$ and $\boldsymbol{\theta}_{\text {res }}$ for the examples considered in this section. We observe there that in most cases the component $\boldsymbol{\theta}_{\text {res }}$ is the very dominant one. The only exception is the situation concerning Example 2 in which $\boldsymbol{\theta}_{\varphi}$ and $\boldsymbol{\theta}_{\text {res }}$ are of the same order. Consequently, any improvement in the a-priori bound for $\hat{\boldsymbol{\sigma}}_{T}$ (even if it could be computed exactly) will not modify the efficiency of the a-posteriori error estimator. In other words, these examples support the belief that $\tilde{\boldsymbol{\theta}}$ will not necessarily yield better results than those given by $\boldsymbol{\theta}$. 
As general remarks, we would like to observe first that the numerical examples presented here behave much better than what the previous theoretical results insinuated. In particular, the order of the constants obtained in Theorems 3.1 and 3.2 indicate that the rates of convergence are affected by large values of $\alpha$, which, nevertheless, was not too severe in the examples. Further, since $\alpha$ is proportional to the inverse of the time-step $\Delta t$, the estimates provided in these theorems also indicate that the convergence of time-dependent solutions should deteriorate as $\Delta t$ decreases. Whether this holds exactly as predicted by the theory or behaves better than that is something to be seen from corresponding numerical results. Now, according to the constants in Theorem 4.1 and Lemma 4.3, one would have expected effectivity indexes between $O\left(\alpha^{-1}\right)$ and $O\left(\frac{\alpha^{3}}{\nu}\right)$. However, as we could see, they all lie on ranges much tighter than that, they do not deteriorate as $N$ increases, and they additionally improve when passing from uniform to adaptive refinements. The above observations yield the conjecture that these constants are overestimated and that they could be improved. In addition, our conclusion is that the proposed mixed method is perhaps not so competitive for extremely large values of $\alpha$, but it does constitute a good alternative for moderately large values of this parameter. Finally, we emphasize that the examples provide enough support for the adaptive algorithm being much more efficient than a uniform refinement when solving the discrete scheme.

\section{References}

[1] D.N. Arnold, J. Douglas JR. And C.P. Gupta, A family of higher order mixed finite element methods for plane elasticity. Numerische Mathematik, 45, (1984), 1-22.

[2] N.S. Bakhvalov, Solution of the Stokes nonstationary problems by the fictitious domain method. Russian Journal of Numerical Analysis and Mathematical Modelling, 10, (1995), 163-172.

[3] R.E. BANK AND A. WeISER, Some a posteriori error estimators for elliptic partial differential equations. Mathematics of Computation, 44, (1985), 283-301.

[4] G.R. Barrenechea and F. Valentin, An unusual stabilized finite element method for a generalized Stokes problem. Numerische Mathematik, 92, 4, (2002), 653-677.

[5] F. Brezzi, M. Fortin, Mixed and Hybrid Finite Element Methods. Springer Verlag, 1991.

[6] U. BRINK AND E. STEIn, A posteriori error estimation in large-strain elasticity using equilibrated local Neumann problems. Computer Methods in Applied Mechanics and Engineering, 161, (1998), 77-101.

[7] J. Cahouet And J.P. Chabard, Some fast 3D finite element solvers for the generalized Stokes problem. International Journal for Numerical Methods in Fluids, 8, (1988), 869-895.

[8] C. Calgaro and J. Laminie, On the domain decomposition method for the generalized Stokes problem with continuous pressure. Numerical Methods for Partial Differential Equations, 16, 1, (2000), 84-106.

[9] C. Carstensen and S. Funken, A-posteriori error control in low-order finite element discretisations of incompressible stationary flows. Mathematics of Computation, 70, 236, (2001), 1353-1381. 
Table 5.1: individual errors, total error e, effectivity index, and global rate of convergence for the uniform refinement (Example 1, $\alpha=10, \nu=1$ ).

\begin{tabular}{|c|c|c|c|c|c|c|c|}
\hline$N$ & $\mathbf{e}(\mathbf{t})$ & $\mathbf{e}(\mathbf{u})$ & $\mathbf{e}(\boldsymbol{\sigma})$ & $\mathbf{e}(p)$ & $\mathbf{e}$ & $\mathbf{e} / \boldsymbol{\theta}$ & $\boldsymbol{\gamma}$ \\
\hline 29 & 3.5762 & 2.4040 & 7.7467 & 3.6114 & 9.5720 & 0.3968 & - \\
105 & 2.2827 & 1.2595 & 4.3739 & 2.0922 & 5.5051 & 0.4343 & 0.8598 \\
401 & 1.3824 & 0.6473 & 2.5351 & 1.2701 & 3.2203 & 0.4912 & 0.8002 \\
1569 & 0.7853 & 0.3244 & 1.2887 & 0.6462 & 1.6735 & 0.5029 & 0.9596 \\
6209 & 0.4206 & 0.1616 & 0.5951 & 0.2791 & 0.7969 & 0.4755 & 1.0787 \\
24705 & 0.2163 & 0.0806 & 0.2776 & 0.1196 & 0.3803 & 0.4525 & 1.0710 \\
98561 & 0.1092 & 0.0402 & 0.1343 & 0.0548 & 0.1860 & 0.4423 & 1.0338 \\
\hline
\end{tabular}

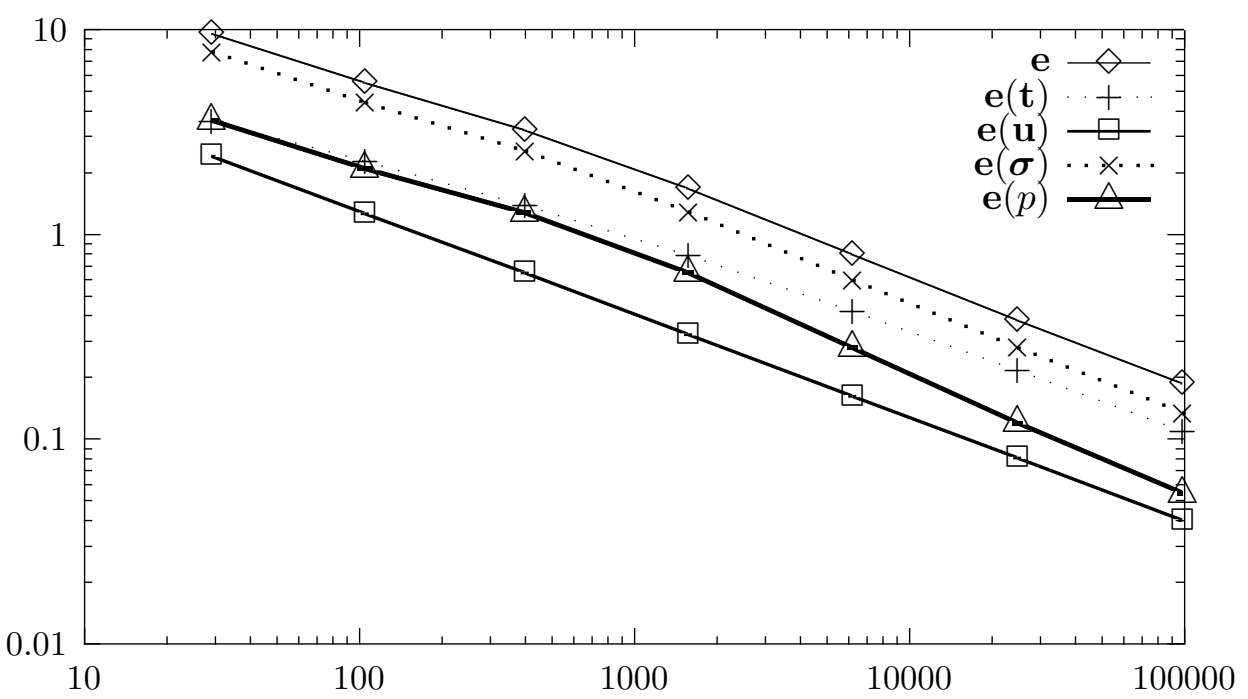

Figure 5.1.1: errors vs. $N$ for the uniform refinement (Example 1, $\alpha=10, \nu=1$ ).

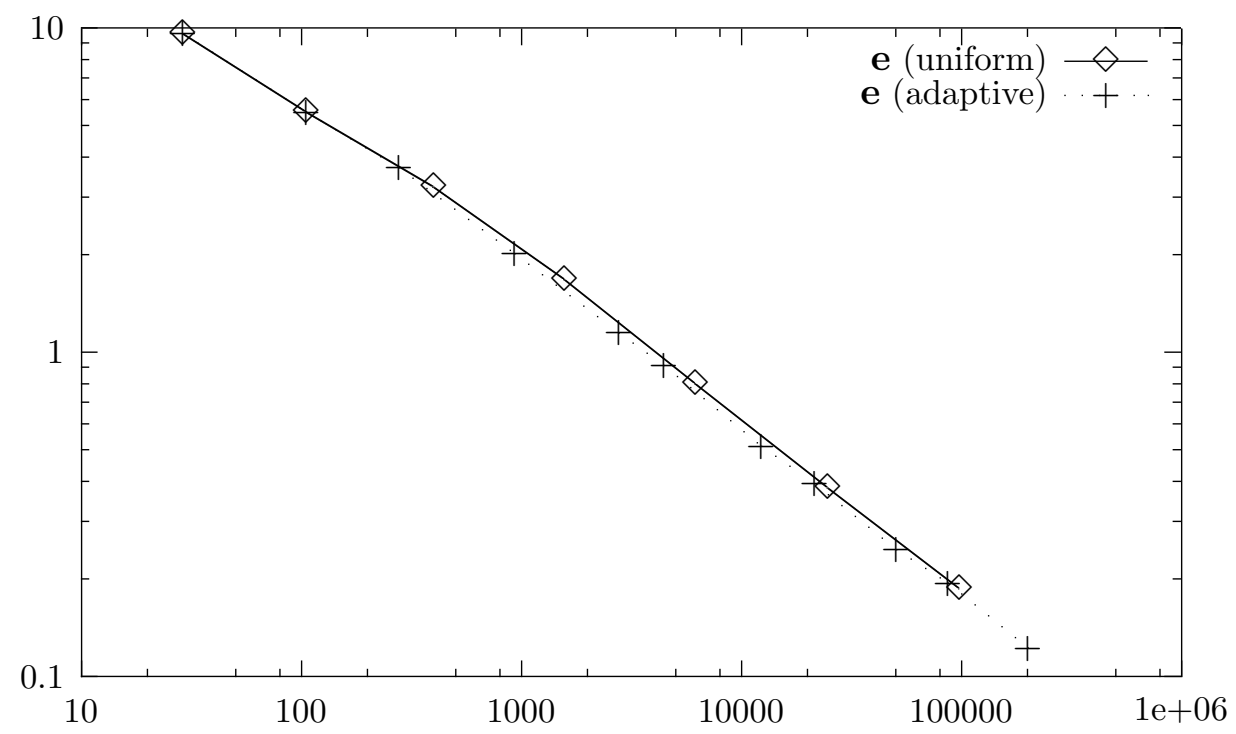

Figure 5.1.2: e vs. $N$ for both refinements (Example 1, $\alpha=10, \nu=1$ ). 
Table 5.2: individual errors, total error e, effectivity index, and global rate of convergence for the uniform refinement (Example 1, $\alpha=100, \nu=1$ ).

\begin{tabular}{|c|c|c|c|c|c|c|c|}
\hline$N$ & $\mathbf{e}(\mathbf{t})$ & $\mathbf{e}(\mathbf{u})$ & $\mathbf{e}(\boldsymbol{\sigma})$ & $\mathbf{e}(p)$ & $\mathbf{e}$ & $\mathbf{e} / \boldsymbol{\theta}$ & $\boldsymbol{\gamma}$ \\
\hline 29 & 3.3494 & 2.3697 & 10.3296 & 4.1105 & 11.8504 & 0.0500 & - \\
105 & 2.0578 & 1.2424 & 7.7385 & 3.1434 & 8.6916 & 0.0700 & 0.4818 \\
401 & 1.1765 & 0.6395 & 5.8418 & 2.3861 & 6.4509 & 0.1011 & 0.4449 \\
1569 & 0.6821 & 0.3224 & 3.3689 & 1.4000 & 3.7254 & 0.1158 & 0.8048 \\
6209 & 0.3909 & 0.1613 & 1.4540 & 0.6076 & 1.6317 & 0.1013 & 1.2003 \\
24705 & 0.2109 & 0.0806 & 0.5048 & 0.2096 & 0.5914 & 0.0734 & 1.4697 \\
98561 & 0.1084 & 0.0402 & 0.1765 & 0.0720 & 0.2230 & 0.0553 & 1.4095 \\
\hline
\end{tabular}

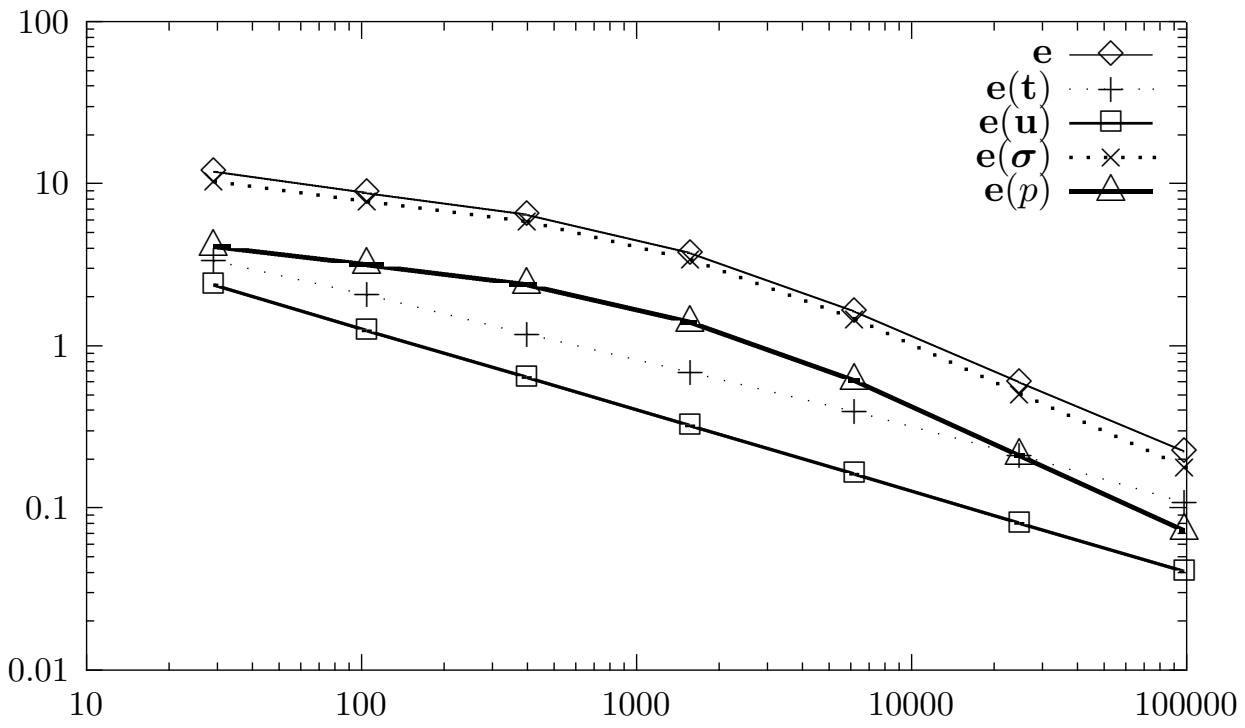

Figure 5.2.1: errors vs. $N$ for the uniform refinement (Example 1, $\alpha=100, \nu=1$ ).

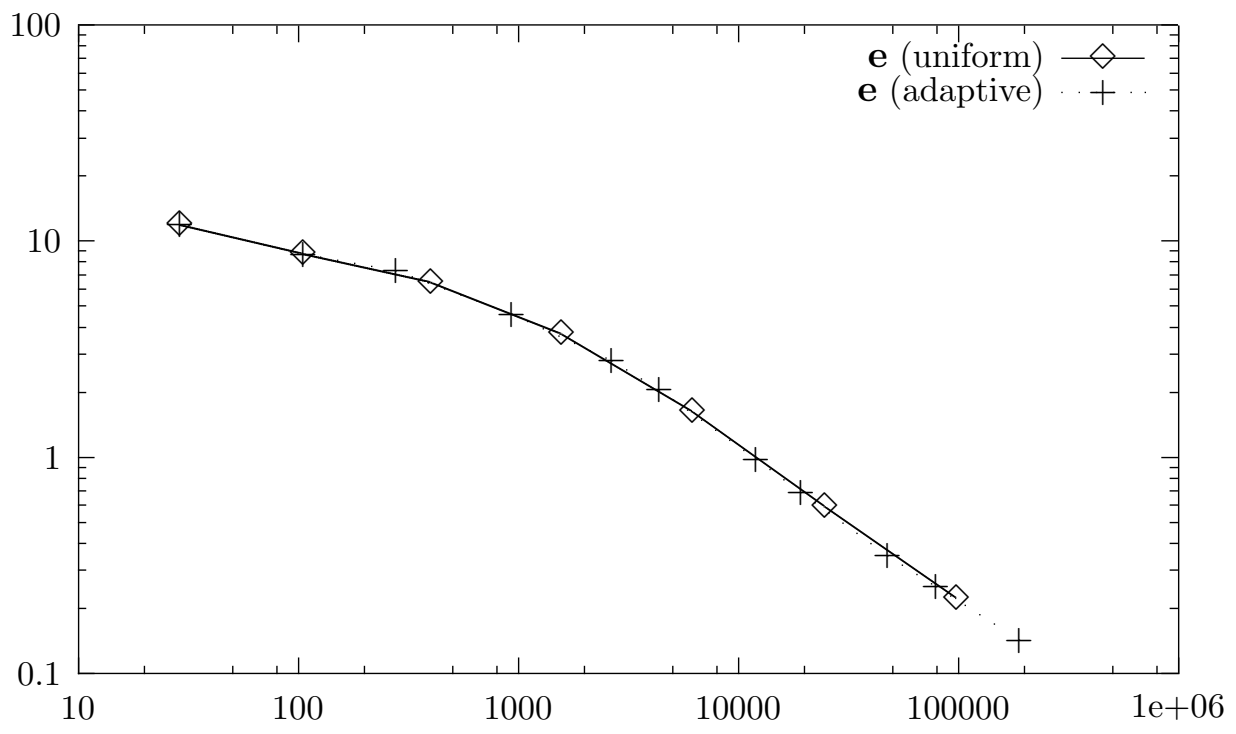

Figure 5.2.2: e vs. $N$ for both refinements (Example 1, $\alpha=100, \nu=1$ ). 
Table 5.3: individual errors, total error e, effectivity index, and global rate of convergence for both refinements (Example 2, $\alpha=100, \nu=0.5$ ).

\begin{tabular}{|c|c|c|c|c|c|c|c|}
\hline$N$ & $\mathbf{e}(\mathbf{t})$ & $\mathbf{e}(\mathbf{u})$ & $\mathbf{e}(\boldsymbol{\sigma})$ & $\mathbf{e}(p)$ & $\mathbf{e}$ & $\mathbf{e} / \boldsymbol{\theta}$ & $\gamma$ \\
\hline 105 & 5.4391 & 0.5032 & 50.7765 & 3.2059 & 51.1700 & 4.5545 & - \\
401 & 4.7510 & 0.5047 & 50.7097 & 2.4017 & 50.9908 & 8.0530 & 0.0052 \\
1569 & 2.7950 & 0.3270 & 32.8015 & 1.1490 & 32.9420 & 10.4012 & 0.6405 \\
6209 & 1.5002 & 0.1760 & 17.6482 & 0.4435 & 17.7182 & 11.1071 & 0.9016 \\
24705 & 0.7768 & 0.0896 & 8.9857 & 0.1716 & 9.0213 & 11.1273 & 0.9775 \\
98561 & 0.3937 & 0.0450 & 4.5126 & 0.0740 & 4.5305 & 11.0640 & 0.9955 \\
\hline 105 & 5.4391 & 0.5032 & 50.7765 & 3.2059 & 51.1700 & 4.5545 & - \\
251 & 4.7575 & 0.5050 & 50.7475 & 2.3963 & 51.0289 & 7.9226 & 0.0063 \\
397 & 2.8246 & 0.3287 & 33.0313 & 1.5115 & 33.1880 & 9.5209 & 1.8766 \\
543 & 1.7132 & 0.1857 & 18.7508 & 1.2146 & 18.8690 & 8.0700 & 3.6061 \\
1105 & 1.2094 & 0.1080 & 10.9389 & 0.9160 & 11.0442 & 7.3584 & 1.5077 \\
2642 & 0.7755 & 0.0624 & 6.3504 & 0.6207 & 6.4279 & 6.5904 & 1.2418 \\
4890 & 0.6248 & 0.0518 & 5.2416 & 0.3865 & 5.2931 & 7.2317 & 0.6310 \\
11810 & 0.4113 & 0.0303 & 3.0674 & 0.2027 & 3.1016 & 6.3483 & 1.2122 \\
26486 & 0.2902 & 0.0217 & 2.2001 & 0.1144 & 2.2222 & 6.4654 & 0.8256 \\
47876 & 0.2133 & 0.0150 & 1.5206 & 0.0723 & 1.5373 & 6.0896 & 1.2448 \\
99636 & 0.1528 & 0.0112 & 1.1353 & 0.0447 & 1.1464 & 6.3580 & 0.8004 \\
190594 & 0.1084 & 0.0075 & 0.7664 & 0.0289 & 0.7746 & 6.0338 & 1.2089 \\
\hline
\end{tabular}

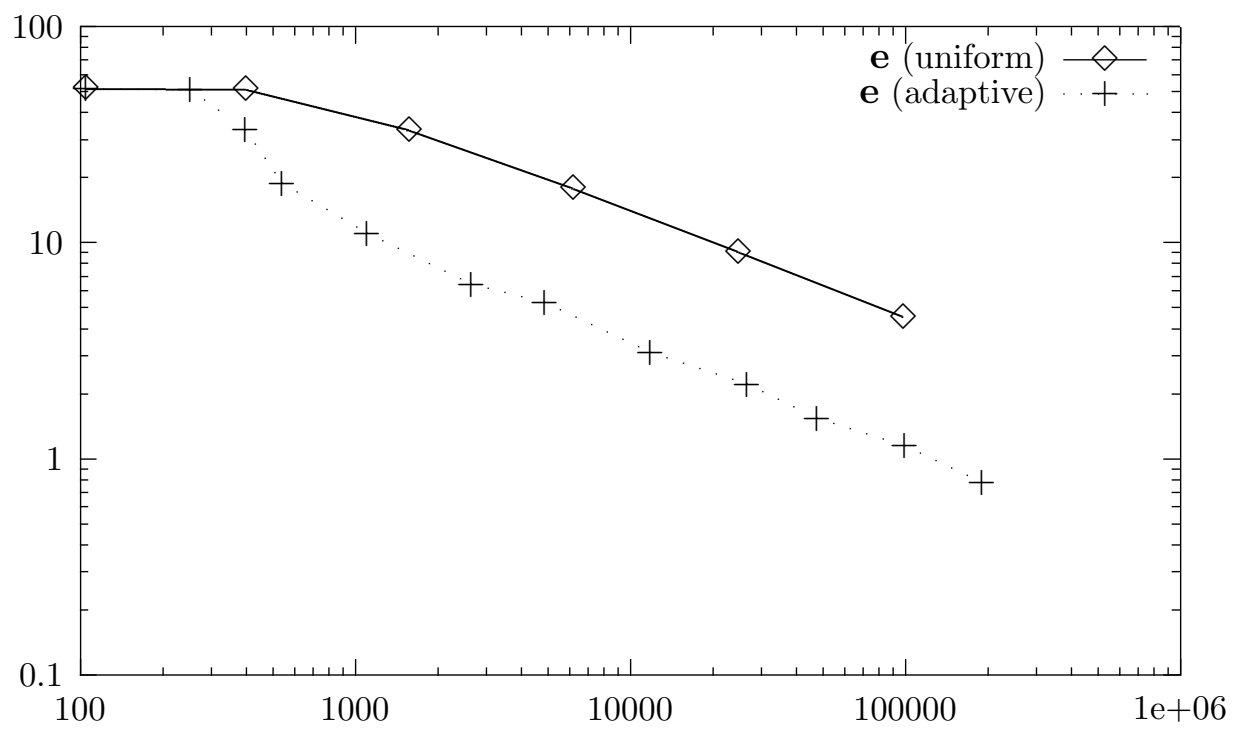

Figure 5.3: e vs. $N$ for both refinements (Example 2, $\alpha=100, \nu=0.5$ ). 
Table 5.4: individual errors, total error e, effectivity index, and global rate of convergence for both refinements (Example 2, $\alpha=1000, \nu=0.5$ ).

\begin{tabular}{|c|c|c|c|c|c|c|c|}
\hline$N$ & $\mathbf{e}(\mathbf{t})$ & $\mathbf{e}(\mathbf{u})$ & $\mathbf{e}(\boldsymbol{\sigma})$ & $\mathbf{e}(p)$ & $\mathbf{e}$ & $\mathbf{e} / \boldsymbol{\theta}$ & $\gamma$ \\
\hline 105 & 7.0383 & 0.0643 & 65.0020 & 5.0629 & 65.5777 & 1.0652 & - \\
401 & 12.9106 & 0.3337 & 334.0185 & 7.4337 & 334.3507 & 7.3412 & - \\
1569 & 17.7197 & 0.5382 & 538.4277 & 8.1264 & 538.7808 & 18.2978 & - \\
6209 & 12.7694 & 0.4502 & 450.3525 & 5.3283 & 450.5652 & 28.8851 & 0.2599 \\
24705 & 7.1160 & 0.2680 & 268.0732 & 2.3559 & 268.1781 & 34.7400 & 0.7514 \\
98561 & 3.7577 & 0.1404 & 140.4863 & 0.9185 & 140.5396 & 36.0464 & 0.9339 \\
\hline 105 & 7.0383 & 0.0643 & 65.0020 & 5.0629 & 65.5777 & 1.0652 & - \\
251 & 12.9142 & 0.3338 & 334.0546 & 6.6592 & 334.3706 & 7.3370 & - \\
397 & 17.7427 & 0.5382 & 538.4458 & 7.0993 & 538.7851 & 18.2613 & - \\
543 & 12.8121 & 0.4501 & 450.2576 & 4.9853 & 450.4676 & 28.6842 & 1.1433 \\
689 & 7.2888 & 0.2693 & 269.4196 & 2.6824 & 269.5316 & 33.3358 & 4.3135 \\
835 & 4.7125 & 0.1543 & 154.4175 & 1.8963 & 154.5011 & 29.2990 & 5.7909 \\
1722 & 2.7302 & 0.0798 & 79.8489 & 1.4523 & 79.9088 & 25.8793 & 1.8217 \\
3839 & 1.6684 & 0.0463 & 46.3600 & 1.3075 & 46.4084 & 24.4862 & 1.3555 \\
7031 & 1.2912 & 0.0363 & 36.4156 & 1.1266 & 36.4560 & 25.8745 & 0.7977 \\
14781 & 0.8664 & 0.0239 & 24.0379 & 1.0019 & 24.0744 & 24.4694 & 1.1169 \\
27310 & 0.6568 & 0.0182 & 18.2701 & 0.7172 & 18.2960 & 25.0696 & 0.8941 \\
56312 & 0.4520 & 0.0123 & 12.3484 & 0.5608 & 12.3694 & 24.2670 & 1.0818 \\
110822 & 0.3375 & 0.0090 & 9.0979 & 0.2983 & 9.1090 & 24.2919 & 0.9038 \\
\hline
\end{tabular}

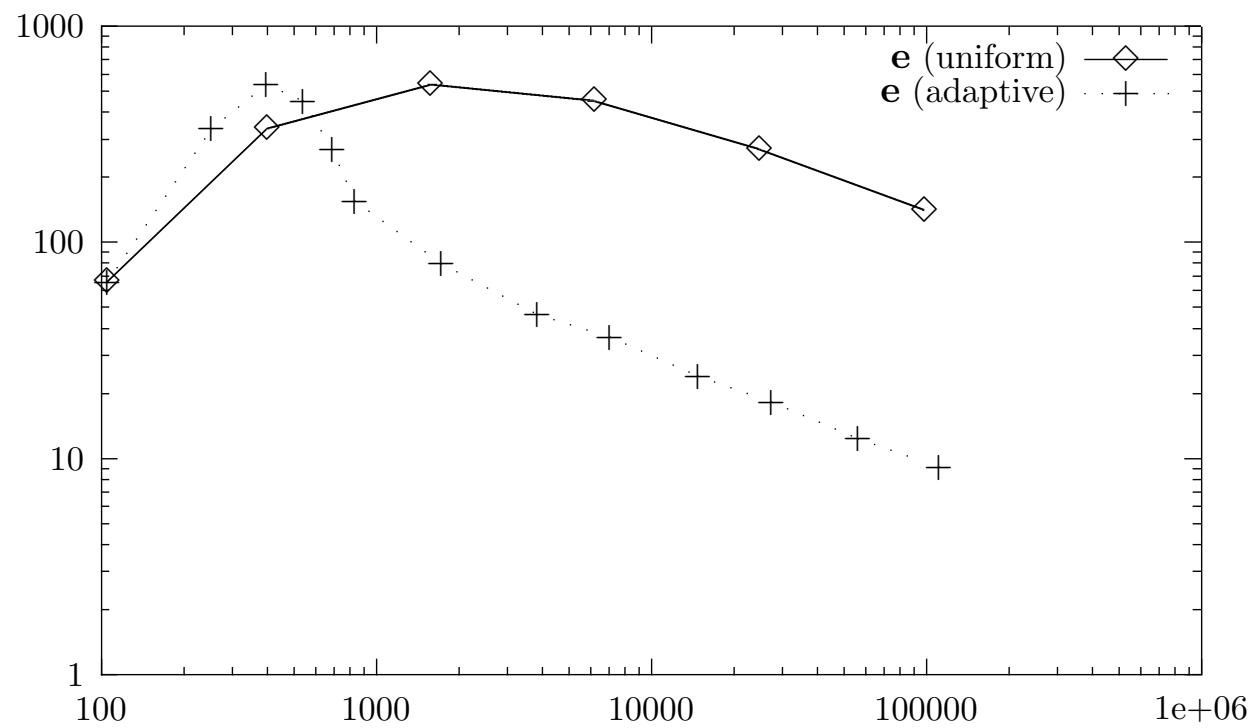

Figure 5.4: e vs. $N$ for both refinements (Example 2, $\alpha=1000, \nu=0.5$ ). 
Meshes 5.3: adapted intermediate meshes with 26486 and 99636 degrees of freedom, respectively, for Example 2, $\alpha=100, \nu=0.5$.

Meshes 5.4: adapted intermediate meshes with 14781 and 56312 degrees of freedom, respectively, for Example 2, $\alpha=1000, \nu=0.5$. 
Table 5.5: individual errors, total error e, effectivity index, and global rate of convergence for both refinements (Example 3, $\alpha=10, \nu=1$ ).

\begin{tabular}{|c|c|c|c|c|c|c|c|}
\hline$N$ & $\mathbf{e}(\mathbf{t})$ & $\mathbf{e}(\mathbf{u})$ & $\mathbf{e}(\boldsymbol{\sigma})$ & $\mathbf{e}(p)$ & $\mathbf{e}$ & $\mathbf{e} / \boldsymbol{\theta}$ & $\boldsymbol{\gamma}$ \\
\hline 29 & 0.7664 & 0.4607 & 6.0365 & 2.8186 & 6.7219 & 1.5756 & - \\
105 & 0.8281 & 0.2984 & 5.1990 & 2.0257 & 5.6487 & 1.8869 & 0.2703 \\
401 & 0.6839 & 0.1733 & 5.3278 & 1.1694 & 5.5001 & 1.3734 & 0.0398 \\
1569 & 0.4615 & 0.0898 & 5.3663 & 0.5403 & 5.4139 & 1.1420 & 0.0231 \\
6209 & 0.2660 & 0.0447 & 4.3239 & 0.2269 & 4.3382 & 1.0762 & 0.3220 \\
24705 & 0.1407 & 0.0223 & 2.7594 & 0.0984 & 2.7649 & 1.0551 & 0.6523 \\
98561 & 0.0718 & 0.0111 & 1.5160 & 0.0454 & 1.5185 & 1.0483 & 0.8662 \\
\hline 29 & 0.7664 & 0.4607 & 6.0365 & 2.8186 & 6.7219 & 1.5756 & - \\
105 & 0.8281 & 0.2984 & 5.1990 & 2.0257 & 5.6487 & 1.8869 & - \\
325 & 0.6916 & 0.1767 & 5.3524 & 1.1959 & 5.5307 & 1.3761 & - \\
471 & 0.5949 & 0.1254 & 5.5517 & 0.9228 & 5.6606 & 1.1705 & \\
617 & 0.5444 & 0.1105 & 4.6251 & 0.8722 & 4.7393 & 1.1340 & 1.3158 \\
763 & 0.5281 & 0.1074 & 3.2728 & 0.8655 & 3.4280 & 1.1900 & 3.0502 \\
909 & 0.5244 & 0.1069 & 2.4193 & 0.8646 & 2.6243 & 1.3368 & 3.0515 \\
1055 & 0.5238 & 0.1069 & 2.1416 & 0.8644 & 2.3706 & 1.4524 & 1.3654 \\
3126 & 0.3502 & 0.0570 & 1.1486 & 0.4108 & 1.2704 & 1.3863 & 1.1485 \\
8157 & 0.2154 & 0.0305 & 0.6736 & 0.2004 & 0.7357 & 1.3126 & 1.1390 \\
12794 & 0.1926 & 0.0258 & 0.5409 & 0.1717 & 0.5998 & 1.3094 & 0.9071 \\
32524 & 0.1147 & 0.0148 & 0.3269 & 0.0905 & 0.3583 & 1.2666 & 1.1042 \\
54229 & 0.0949 & 0.0117 & 0.2551 & 0.0726 & 0.2819 & 1.2478 & 0.9378 \\
130544 & 0.0578 & 0.0071 & 0.1621 & 0.0423 & 0.1774 & 1.2398 & 1.0541 \\
\hline
\end{tabular}

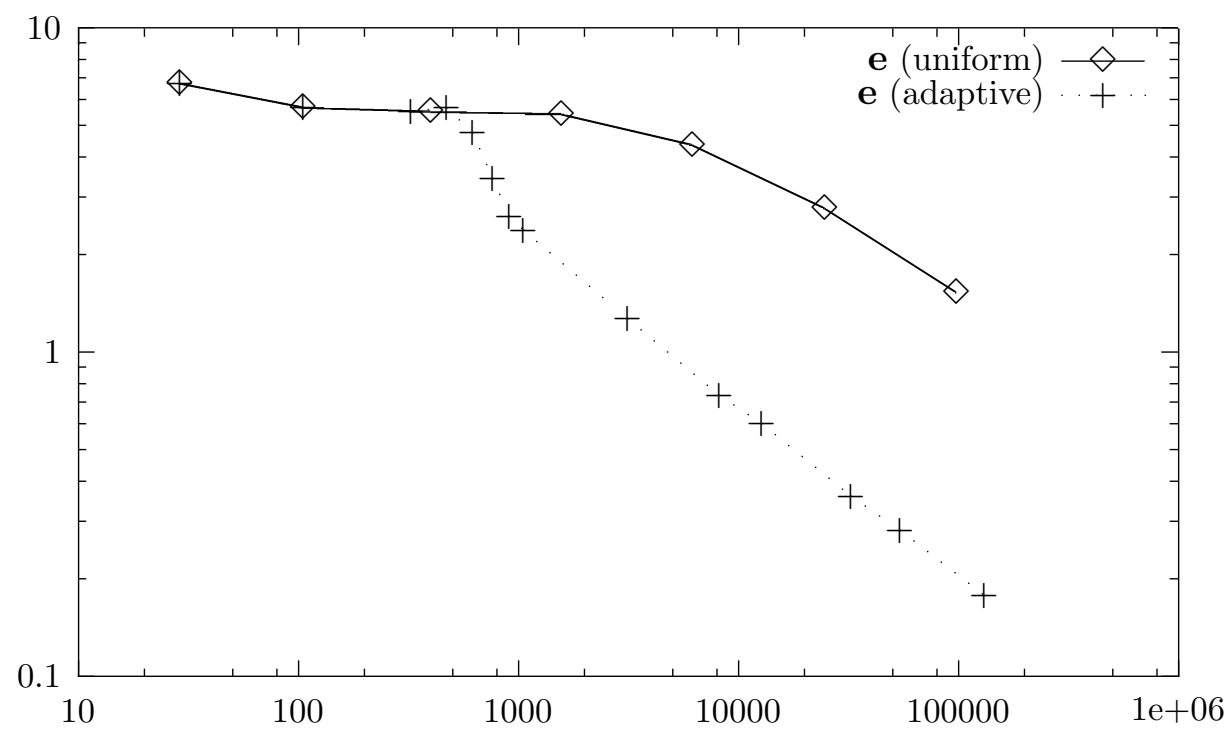

Figure 5.5: e vs. $N$ for both refinements (Example 3, $\alpha=10, \nu=1$ ). 
Table 5.6: individual errors, total error e, effectivity index, and global rate of convergence for both refinements (Example 3, $\alpha=100, \nu=1$ ).

\begin{tabular}{|c|c|c|c|c|c|c|c|}
\hline$N$ & $\mathbf{e}(\mathbf{t})$ & $\mathbf{e}(\mathbf{u})$ & $\mathbf{e}(\boldsymbol{\sigma})$ & $\mathbf{e}(p)$ & $\mathbf{e}$ & $\mathbf{e} / \boldsymbol{\theta}$ & $\boldsymbol{\gamma}$ \\
\hline 29 & 0.3670 & 0.3899 & 7.2973 & 2.9958 & 7.9065 & 0.2145 & - \\
105 & 0.4457 & 0.2537 & 7.0634 & 2.8054 & 7.6175 & 0.3444 & 0.0578 \\
401 & 0.4376 & 0.1525 & 6.7047 & 2.1753 & 7.0639 & 0.6181 & 0.1125 \\
1569 & 0.3580 & 0.0849 & 5.9249 & 1.2798 & 6.0727 & 1.0757 & 0.2216 \\
6209 & 0.2400 & 0.0441 & 4.4515 & 0.5381 & 4.4906 & 1.3042 & 0.4388 \\
24705 & 0.1363 & 0.0222 & 2.7790 & 0.1799 & 2.7883 & 1.2948 & 0.6901 \\
98561 & 0.0712 & 0.0111 & 1.5186 & 0.0607 & 1.5215 & 1.2738 & 0.8754 \\
\hline 29 & 0.3670 & 0.3899 & 7.2973 & 2.9958 & 7.9065 & 0.2145 & \\
105 & 0.4457 & 0.2537 & 7.0634 & 2.8054 & 7.6175 & 0.3444 & 0.0578 \\
251 & 0.4305 & 0.1595 & 7.1218 & 2.4020 & 7.5300 & 0.6149 & 0.0264 \\
789 & 0.3678 & 0.0912 & 6.4211 & 1.7284 & 6.6605 & 1.0505 & 0.2142 \\
2483 & 0.2812 & 0.0501 & 4.7966 & 0.9914 & 4.9063 & 1.2275 & 0.5332 \\
2629 & 0.2520 & 0.0431 & 3.4981 & 0.9790 & 3.6415 & 0.9561 & 10.4354 \\
2947 & 0.2383 & 0.0403 & 2.6186 & 0.9350 & 2.7910 & 0.8150 & 4.6587 \\
4633 & 0.2129 & 0.0327 & 1.9695 & 0.7235 & 2.1093 & 0.7499 & 1.2380 \\
11357 & 0.1568 & 0.0214 & 1.3188 & 0.3226 & 1.3669 & 0.7916 & 0.9675 \\
22547 & 0.1249 & 0.0147 & 0.7832 & 0.2293 & 0.8257 & 0.6380 & 1.4699 \\
46839 & 0.0819 & 0.0101 & 0.5382 & 0.1011 & 0.5538 & 0.6511 & 1.0928 \\
89728 & 0.0673 & 0.0074 & 0.3716 & 0.0744 & 0.3850 & 0.5881 & 1.1184 \\
187253 & 0.0423 & 0.0051 & 0.2704 & 0.0362 & 0.2761 & 0.6391 & 0.9030 \\
\hline
\end{tabular}

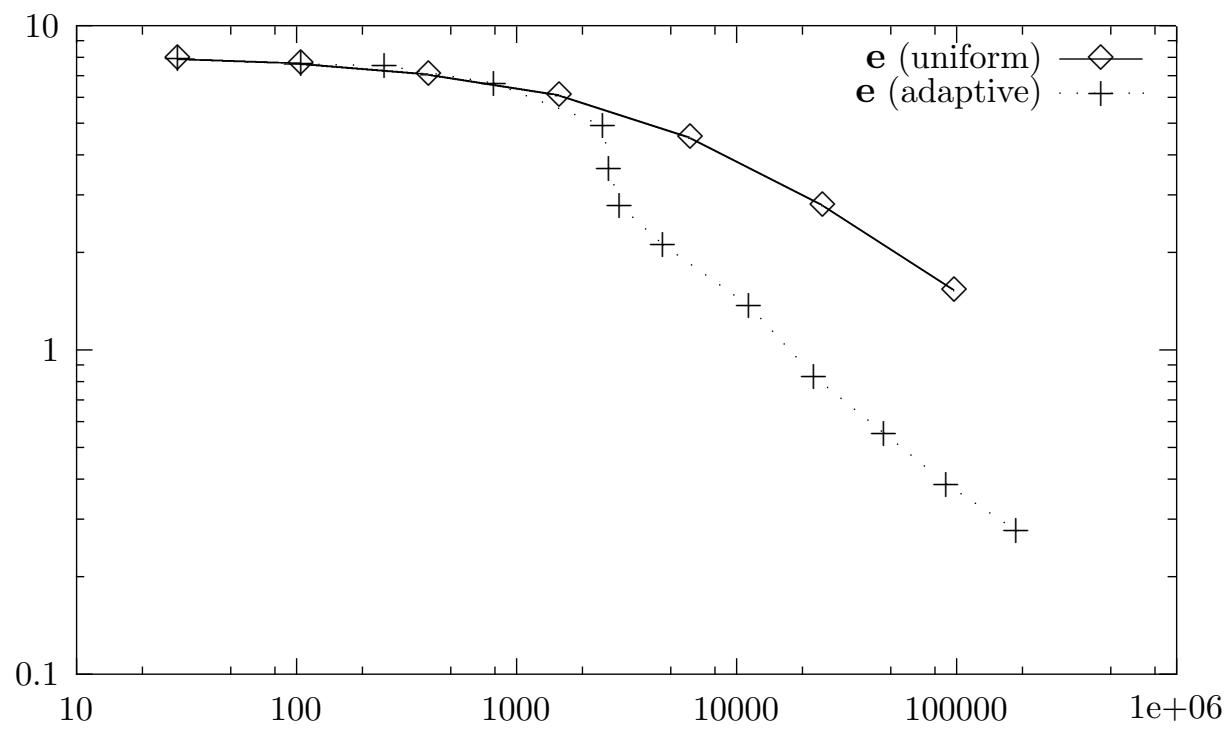

Figure 5.6: e vs. $N$ for both refinements (Example 3, $\alpha=100, \nu=1$ ). 
Meshes 5.5: adapted intermediate meshes with 3126 and 54229 degrees of freedom, respectively, for Example $3, \alpha=10, \nu=1$.

Meshes 5.6: adapted intermediate meshes with 4633 and 46839 degrees of freedom, respectively, for Example 3, $\alpha=100, \nu=1$. 
Table 5.7: individual errors, total error e, effectivity index, and global rate of convergence for both refinements (Example 4, $\alpha=1000, \nu=0.5$ ).

\begin{tabular}{|c|c|c|c|c|c|c|c|}
\hline$N$ & $\mathbf{e}(\mathbf{t})$ & $\mathbf{e}(\mathbf{u})$ & $\mathbf{e}(\boldsymbol{\sigma})$ & $\mathbf{e}(p)$ & $\mathbf{e}$ & $\mathbf{e} / \boldsymbol{\theta}$ & $\boldsymbol{\gamma}$ \\
\hline 105 & 74.3233 & 10.9499 & 975.4179 & 26.3889 & 978.6626 & 0.0838 & - \\
401 & 43.9757 & 7.2191 & 790.4335 & 25.1620 & 792.0885 & 0.1017 & 0.3156 \\
1569 & 32.5849 & 4.0695 & 513.2866 & 11.7287 & 514.4697 & 0.1160 & 0.6326 \\
6209 & 17.3618 & 2.1141 & 264.9580 & 5.2669 & 265.5869 & 0.1147 & 0.9613 \\
24705 & 8.9221 & 1.0675 & 132.0790 & 2.1941 & 132.4025 & 0.1130 & 1.0080 \\
98561 & 4.5016 & 0.5350 & 65.7869 & 0.8889 & 65.9489 & 0.1123 & 1.0074 \\
\hline 105 & 74.3233 & 10.9499 & 975.4179 & 26.3889 & 978.6626 & 0.0838 & - \\
251 & 47.5896 & 7.5069 & 785.4328 & 25.8358 & 787.3331 & 0.0978 & 0.4992 \\
907 & 34.5913 & 4.2248 & 536.2225 & 13.2819 & 537.5178 & 0.1164 & 0.5942 \\
2111 & 20.9361 & 2.1538 & 292.7591 & 8.1437 & 293.6276 & 0.1247 & 1.4315 \\
7141 & 11.2381 & 1.1037 & 148.3617 & 4.1104 & 148.8476 & 0.1240 & 1.1149 \\
17599 & 9.4008 & 0.6518 & 91.1181 & 3.1416 & 91.6579 & 0.1334 & 1.0750 \\
30105 & 5.9042 & 0.5120 & 68.4031 & 2.1752 & 68.6938 & 0.1244 & 1.0744 \\
87607 & 4.1128 & 0.2886 & 41.6759 & 1.6638 & 41.9124 & 0.1367 & 0.9250 \\
120853 & 3.5184 & 0.2520 & 33.8690 & 1.3457 & 34.0788 & 0.1243 & 1.2862 \\
\hline
\end{tabular}

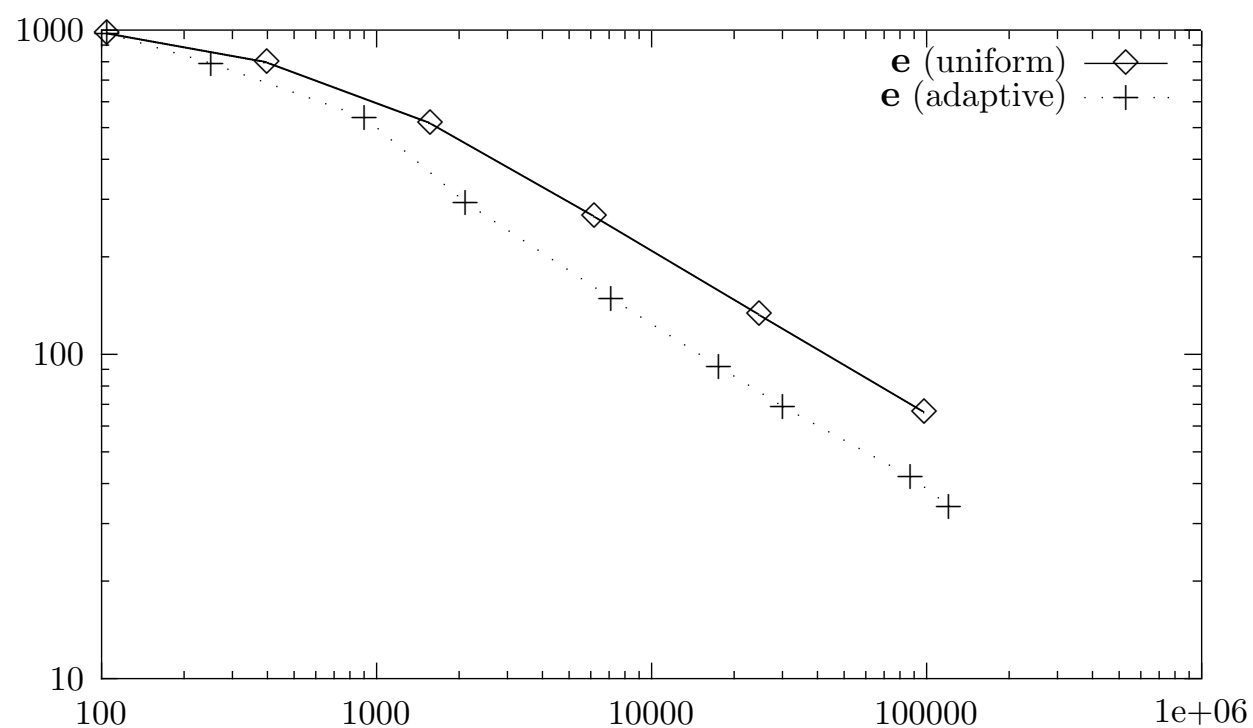

Figure 5.7: e vs. $N$ for both refinements (Example 4, $\alpha=1000, \nu=0.5$ ). 
Meshes 5.7: adapted intermediate meshes with 7141, 17599, 30105 and 87607 degrees of freedom, respectively, for Example 4, $\alpha=1000, \nu=0.5$. 
Table 5.8: Main components of the a-posteriori error estimate $\boldsymbol{\theta}$.

\begin{tabular}{|c|c|c|c|c|c|}
\hline \multicolumn{3}{|c|}{ Example $1(\alpha=10, \quad \nu=1)$} & \multicolumn{3}{|c|}{ Example $1(\alpha=100, \quad \nu=1)$} \\
\hline$N$ & $\boldsymbol{\theta}_{\varphi}$ & $\boldsymbol{\theta}_{\text {res }}$ & $N$ & $\boldsymbol{\theta}_{\varphi}$ & $\boldsymbol{\theta}_{\text {res }}$ \\
\hline 29 & 4.5135 & 23.6942 & 29 & 4.3964 & 236.8632 \\
\hline 105 & 2.5688 & 12.4118 & 105 & 2.4076 & 124.1070 \\
\hline 401 & 1.5104 & 6.3792 & 401 & 1.3361 & 63.7909 \\
\hline 1569 & 0.8615 & 3.2136 & 1569 & 0.7612 & 32.1361 \\
\hline 6209 & 0.4659 & 1.6099 & 6209 & 0.4345 & 16.0988 \\
\hline 24705 & 0.2404 & 0.8053 & 24705 & 0.2346 & 8.0533 \\
\hline 98561 & 0.1213 & 0.4027 & 98561 & 0.1205 & 4.0271 \\
\hline \multicolumn{3}{|c|}{ Example $2(\alpha=1000, \quad \nu=0.5)$} & \multicolumn{3}{|c|}{ Example $3(\alpha=10, \quad \nu=1)$} \\
\hline$N$ & $\boldsymbol{\theta}_{\varphi}$ & $\boldsymbol{\theta}_{\text {res }}$ & $N$ & $\boldsymbol{\theta}_{\varphi}$ & $\boldsymbol{\theta}_{\text {res }}$ \\
\hline 105 & 61.5259 & 2.1109 & 29 & 1.9781 & 3.7799 \\
\hline 251 & 45.5072 & 2.4457 & 105 & 1.3309 & 2.6815 \\
\hline 397 & 29.3608 & 2.9050 & 325 & 0.8823 & 3.9209 \\
\hline 543 & 15.4992 & 2.5303 & 471 & 0.6377 & 4.7937 \\
\hline 689 & 7.8532 & 1.9235 & 617 & 0.5360 & 4.1447 \\
\hline 835 & 4.9966 & 1.6857 & 763 & 0.5107 & 2.8349 \\
\hline 1722 & 2.8452 & 1.1997 & 909 & 0.5060 & 1.8968 \\
\hline 3839 & 1.7123 & 0.8124 & 1055 & 0.5053 & 1.5520 \\
\hline 7031 & 1.3031 & 0.5357 & 3126 & 0.3455 & 0.8487 \\
\hline 14781 & 0.8945 & 0.4096 & 8157 & 0.2140 & 0.5180 \\
\hline 27310 & 0.6765 & 0.2737 & 12794 & 0.1890 & 0.4173 \\
\hline 56312 & 0.4681 & 0.2018 & 32524 & 0.1141 & 0.2589 \\
\hline 110822 & 0.3512 & 0.1313 & 54229 & 0.0942 & 0.2054 \\
\hline & & & 130544 & 0.0576 & 0.1310 \\
\hline \multicolumn{3}{|c|}{ Example $3(\alpha=100, \quad \nu=1)$} & \multicolumn{3}{|c|}{ Example $4(\alpha=1000, \quad \nu=0.5)$} \\
\hline$N$ & $\boldsymbol{\theta}_{\varphi}$ & $\boldsymbol{\theta}_{\text {res }}$ & $N$ & $\boldsymbol{\theta}_{\varphi}$ & $\boldsymbol{\theta}_{\text {res }}$ \\
\hline 29 & 1.7393 & 36.8189 & 105 & 75.8505 & 11672.9488 \\
\hline 105 & 1.1333 & 22.0862 & 251 & 55.7055 & 8048.8130 \\
\hline 251 & 0.7085 & 12.2245 & 907 & 34.1669 & 4614.5160 \\
\hline 789 & 0.4471 & 6.3244 & 2111 & 20.4605 & 2353.0475 \\
\hline 2483 & 0.2940 & 3.9861 & 7141 & 11.3901 & 1199.6719 \\
\hline 2629 & 0.2491 & 3.8005 & 17599 & 9.3005 & 687.0050 \\
\hline 2947 & 0.2356 & 3.4162 & 30105 & 5.9360 & 552.0031 \\
\hline 4633 & 0.2104 & 2.8048 & 87607 & 4.1046 & 306.5056 \\
\hline 11357 & 0.1580 & 1.7195 & 120853 & 3.5123 & 274.0432 \\
\hline 22547 & 0.1249 & 1.2881 & & & \\
\hline 46839 & 0.0824 & 0.8465 & & & \\
\hline 89728 & 0.0680 & 0.6510 & & & \\
\hline 187253 & 0.0427 & 0.4300 & & & \\
\hline
\end{tabular}


[10] G.N. Gatica, An application of Babuška-Brezzi's theory to a class of variational problems. Applicable Analysis, 75, 3-4, (2000), 297-303.

[11] G.N. Gatica, Solvability and Galerkin approximations of a class of nonlinear operator equations. Zeitschrift für Analysis und ihre Anwendungen, 21, 3, (2002), 761-781.

[12] G.N. Gatica, M. González and S. Meddahi, A low-order mixed finite element method for a class of quasi-Newtonian Stokes flows. Part I: a-priori error analysis. Computer Methods in Applied Mechanics and Engineering, vol. 193, 9-11, pp. 881-892, (2004).

[13] G.N. Gatica, M. González and S. Meddahi, A low-order mixed finite element method for a class of quasi-Newtonian Stokes flows. Part II: a-posteriori error analysis. Computer Methods in Applied Mechanics and Engineering, vol. 193, 9-11, pp. 893-911, (2004).

[14] G.N. Gatica, N. Heuer and S. Meddahi, On the numerical analysis of nonlinear twofold saddle point problems. IMA Journal of Numerical Analysis, 23, 2, (2003), 301-330.

[15] G.N. Gatica And E.P. Stephan, A mixed-FEM formulation for nonlinear incompressible elasticity in the plane. Numerical Methods for Partial Differential Equations, 18, 1, (2002), 105-128.

[16] G.M. Kobelkov and M.A. OlshanskiI, Effective preconditioning of Uzawa type schemes for a generalized Stokes problem. Numerische Mathematik, 86, (2000), 443-470.

[17] O. Ladyzhenskaya, New equations for the description of the viscous incompressible fluids and solvability in the large for the boundary value problems of them. In Boundary Value Problems of Mathematical Physics V, AMS (Providence), 1970.

[18] J.L. Lions and E. Magenes, Problèmes aux Limites non Homogènes et Applications. I. Dunod, Paris, 1968.

[19] B.V. PAL'TSEV, On rapidly converging iterative methods with incomplete splitting of boundary conditions for a multidimensional singularly perturbed system of Stokes type. Russian Academy of Sciences, Sbornik Mathematics, 81, (1995), 487-531.

[20] J.E. Roberts and J.-M. Thomas, Mixed and Hybrid Methods. In Handbook of Numerical Analysis, vol. II, edited by P.G. Ciarlet y J.L. Lions, North-Holland (Amsterdam), 1991.

[21] V. Sarin And A. Sameh, An efficient iterative method for the generalized Stokes problem. SIAM Journal on Scientific Computing, 19, 1, (1998), 206-226.

[22] B. Scheurer, Existence et approximation de point selles pou certain problemes nonlinéaires. RAIRO Analyse Numérique, 11, 4, (1977), 369-400.

[23] R. Verfürth, A Review of A Posteriori Error Estimation and Adaptive Mesh-Refinement Techniques. Wiley-Teubner (Chichester), 1996. 